Aus der gynäkologischen Abtheilung des Krankenhauses der Elisabethinerinnen in Breslau (Prof. Pfannenstiel).

\title{
Ein Beitrag zur Lehre von den Tuboovarialcysten.
}

\author{
Von
}

Georg Preiser, prakt. Arzt.

(Mit 7 Abbildungen im Text.)

Als Tuboovarialcysten fasst man eystische Gebilde am $\Lambda$ dnexapparat des Uterus auf, deren Wandung z. Th. vom Eierstock, z. Th. vom Eileiter gebildet wird.

Seitdem Burnier $1880^{1}$ ) durch eine von vielen Forschern angenommene Entstchungsthcorie der Tuboovarialcysten die Aufmerksamkeit auf diese Art von Adnextumoren gelenkt hatte, häufte sich fast mit jeder neuen Veröffentlichung von Fällen auch eine Entstehungstheorie auf die andere. Wie Zahn und Orthmann richtig sagen, krankte jede derselben daran, dass sie alle Fälle auf ihre Weise entstanden wissen wollte. Heute steht nun fest, dass mehrere Entstehungsweisen möglich sind, wofür auch die Verschiedenheit der pathologisch-anatomischen Befunde spricht.

Aufgabe dieser Arbeit soll es sein, an der Hand der Literatur und unter Beibringung einiger neuer Fälle einmal die vorliegenden Entstehungstheorien einer kritischen .Beleuchtung zu unterziehen und zugleich eine neue Erklärung für diejenige Gruppe von Tuboovarialcysten zu bringen, bei der die Fimbrien des Eileiters in der Ovarialcyste frei flottiren oder sich fächerförmig ausgebreitet an ihrer Wand angewachsen finden. Und zwar werde ich versuchen, hier eine ausführlichere Beschreibung derjenigen Morphogenese von

1) Burnier, Zeitschr. für Geb. und Gyn. V u. VI. Ueber Tuboovarialcysten. 
Tuboovarialcysten zu bringen, die zum ersten Mal von meinem verehrten Lehrer, Herrn Prof: Dr. Pfannenstiel-Breslau, in den Verhandlungen der Deutschen Gesellschaft für Gynäkologie 1899 hervorgehoben wurde. Seiner Güte verdanke ich auch das Material zur vorliegenden Arbeit, wofür es mit gestattet sei, ihm auch an dieser Stelle ergebenst zu danken. Meinen Dank auch seinem ersten Assistenten, Herrn Dr. Kroemer, für die freundliche Unterstützung bei der Anfertigung der mikroskopischən Präparate und die Ueberlassung zweier Zeichnungen.

Von vornherein möchte ich erwähnen, dass ich in dieser Arbeit nur echte Tuboovarialcysten bespreche, nicht aber Tuboovarialneubildungen. Auch die eigentlichen Tuboovarialabscesse sollon nur soweit Berücksichtigung finden, als sie genetisch mit den Tuboovarialcysten in Verbindung zu bringen sind.

Wenn wir zunächst die Verschiedenheiten im anatomischen Bau der Tuboovarialcyste ins Auge fassen, so tritt uns als häufigste Form ein Adnextumor von typischer Retortengestalt entgegen. Auf dem Durchschnitt zeigt er die meist stark erweiterte Tube, deren Fimbrien in der Regel vollständig fehlen. Die Wand der Tube geht unmittelbar in die Wand der mit der Tube communicirenden Ovarialcyste über. In anderen Fällen jedoch ist zwischen Tubar- und Ovarialcyste eine deutliche Zwischencyste eingeschoben, ja man kann sogar das Ovarium oft noch völlig unverändert an dieser erwähnten Zwischencyste angelöthet finden. In diesen Fällen finden sich die Fimbrien häufig gut erhalten im Inneren der Tubỏovarialcyste, entweder frei flottirend oder an ihrer Wand festgewachsen. In den meisten Fällen finden wir deutliche Entzündungserscheinungen oder deren Ueberbleibsel ${ }^{1}$ ); doch ist auch zuweilen die Tuboovarialcyste an ihrer Aussenfläche so glatt, dass man selbst an eine congenitale Verbildung gedacht und den ganzen Befuud als "Ovarialtube" bezeichnet hat.

Reboul2), der das erste Mal in der Literatur eine morphogenetische Eintheilung der Tuboovarialcysten giebt, nimmt eine intrauterine Entstehungsweise als möglich an, indem er dem Beispiele Beaucamp's ${ }^{8}$ ) folgt. Beim Menschen ist noch nie ein Fall

1) v. Rosthorn, Billroth-Festschrift 1892. Beitr. zur Kenntniss der Tuboovarialcysten. S. 10.

2) Reboul, Inaug.-Dissert. Berlin 1895. Ueber Tuboovarialcysten.

3) E. Beaucamp, Ueber Tuboovarialschwangerschaft. Inaug.-Dissert. Berlin 1884. 
beobachtet worden, der mit Sicherheit als congenital bezeichnet werden könnte.

Jedoch beschreibt Schneidemühl1) eine "Ovarialtube" beim Pferde, die er deswegen für congenital anspricht, weil in ihrer Umgebung keine makroskopischen Entzündungserscheinungen zu sehen sind. Er hält es „auf Grund entwickelungsgeschichtlicher Erwägungen " für ${ }^{2}$ ) "leicht denkbar, dass gelegentlich keine freie Bauchöffnung an der Tube entsteht, sondern diese innerhalb des Eierstocks endigt, oder dass schon um dieselbe Zeit eine vollständige Vereinigung derselben mit der Oberfläche des Ovariums eintritt, so dass die Fimbriae tubae vollständig fehlen. Durch dieses vitium primae formationis würde dann die Erklärung gefunden sein für das beobachtete Vorkommen einer Verbindung des Eierstocks mit der Tube, wie auch andererseits der Boden geschaffen sein würde, um bei Erkrankungen des Ovariums, die zur Cystenbildung führen, die Tuboovarialcysten zur Entstehung zu bringen".

$\mathrm{Zahn}^{3}$ ) sagt nun in seiner Kritik dieses Berichtes: "Die Abwesenheit von sonstigen makroskopischen peritonitischen Veränderungen würde nicht sehr beweiskräftig gegen die Annahme eines später stattgehabten entzündlichen Processes an den Tubenostien sprechen, da ja, wie Jeder weiss, der öfter bei Thieren operative Eingriffe an den Bauchorganen vornahm, entzündliche Vorgänge am Bauchfell so glatt ablaufen können, dass oft nach nicht vielen Wochen kaum eine oder selbst keine Spur von Entzündungsresten aufgefunden werden kann."

Eine recht interessante Erklärung für die sogenannte "congenitale Ovarialtube" glaubt $\mathrm{Zedel}^{4}$ ) auf Grund der entwickelungsgeschichtlichen Thatsache gefunden $z u$ haben, dass das proximale Ende des Müller'schen Ganges zuerst als eine Epithelrinne angelegt werde; der obere Theil der Rinne bleibe normaler Weise offen und werde zum abdominalen Ostium der Tube. Nun wäre es aber denkbar, dass sich auch dieser Theil ebenso wie der untere schliesse und sich nichtsdestoweniger in allen seinen Theilen vollkommen ausbilde,' also auch die Tubenfranzen. Entstehe nun ein Hydro-

1) G. Schneidemühl, Deutsch. Zeitschr. für Thiermedicin und vergl. Pathol. 1883. Bd. 9. S. 279.

2) Citirt nach F. W. Zahn, Ueber Tuboovarialcysten. Virchow's Arch. 151. S. 268.

3) F. W. Zahn, Ueber Tuboovarialcysten. Virch. Arch. 151. S. 276.

4) Zedel, Zeitschr. für Geburtsh. und Gyn. 1894. 
salpinx, so könne dieser leicht mit dem Ovarium verschmelzen zur Ovarialtube.

Zedel sagt aber selbst: „dass die Erklärung der Bildung durch einen Entwickelungsfehler manche Bedenken gegen sich hat".

Alle Autoren, mit Ausnahme der oben erwähnten Reboul und Beaucamp, sprechen sich auch gegen die congenitale "Ovarialtube" beim Menschen aus.

Was nun die extrauterine Genese der Tuboovarialcyste anbebelangt, so will ich hier mehrere Theorien, die nur zum Theil einwandsfrei bewiesen sind, anführen. Alle haben zunächst ein Gemeinsames in der Annahme eines entzündlichen Vorganges an den Adnexen als Vorbedingung zum Zustandekommen einer Tuboovarialcyste. Als solcher kommt wohl vorzugsweise Gonorrhoe in Betracht. Bei Beantwortung der Frage: Wie sind Ovarium und Tube mit einander verwachsen, oder wie konnten Tubarcyste und Ovarialcyste mit einander in Verbindung treten? gehen aber die Meinungen völlig auseinander.

Zunächst sei es mir gestattet, an eine längst von mehreren Autoren sicher bewiesene Form des Zustandekommens der Tuboovarialcysten $\mathrm{zu}$ erinnern, die in den neueren Arbeiten nicht scharf genug betont und zum Theil gar nicht erwähnt wird: Es ist dies eine Entstehungsweise via Hydro-oder Pyosalpinx.

Rokitansky ${ }^{1}$, Virchow ${ }^{2}$ ), Klob ${ }^{3}$ ), Klebs ${ }^{4}$ ), Orth ${ }^{5}$ ), Burnier ${ }^{6}$ ), Orthmann ${ }^{7}$ ), v. Rosthorn ${ }^{8}$ ) und Andere haben gezeigt, dass der Tubenverschluss am abdominalen Ende nicht durch Verwachsung der epithelisirten Innenfläche der Fimbrien aneinander möglich ist, sondern dass die Fimbrien sich vielmehr einkrempeln und mit ihren peritonealen Flächen mit einander verkleben, sodass, wenn die Tube secernirt und das Secret nicht entleert werden kann, ein Pyo- oder Hydrosalpinx entsteht. Wird dieser Sactosalpinx jetzt so gross, dass er das Ovarium berührt, so ist die

1) Rokitansky, Ueber Abnormitäteu des Corpus luteum. Allgemeine Wiener med. Ztg. Jg. 1859. No. 34 und 35.

2) Virchow, Die krankhaften Geschwülste. Berlin 1863. Bd. 1.

3) Klob, Patholog. Anat. der weibl. Sexualorgane. Wien 1864. S. 347.

4) Klebs, Hdbch. der path. Anat. Berlin 1876. Bd. 1. 2. Abth.

5) Orth, Lehrb. der spec. path. Anat. Berlin 1893. Bd. 2. 1. Abth.

6) Burnier, Ueber Tuboovarialcysten. Ztschr. für Geb. u. Gyn. Bd. 5 .

7) Orthmann, Virchow's Archiv. 155. S. 224.

8) v. Rosthorn, Beitr. zur Kenntniss der Tuboovarialcysten. BillrothFestschrift. 1892. 
Figur 1.
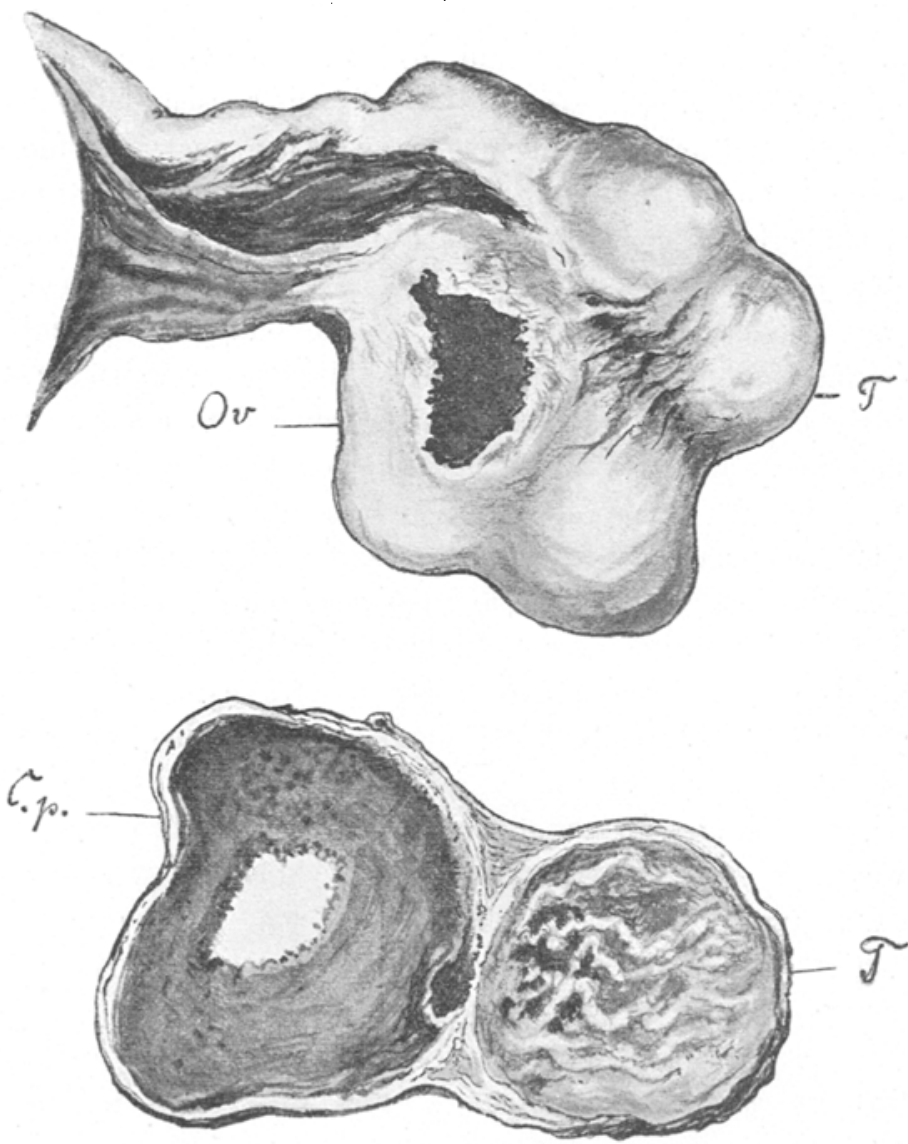

Rechtsseitiger Adnextumor, veranschaulicht das Entstehen einer Tuboovarialcyste aus einer Corp.-lut.-Cyste und einer mit ihr verwachsenen Sactosalpinx. Man sieht auf dem Durchschnitt, wie sich durch Druckatrophie der Zwischenwand der Durchbruch vorbereitet. Die Oeffnung in der Wand der Corp.-lut.Cyste entspricht der Verwachsungsstelle des Adnextumors mit dem Rectum. T. Tube. - Ov. Ovarium. - C. p. Corp.-lut.-Cyste. (Der Durchschnitt entspricht nicht dem Profil der oberen Zeichnung.)

Grundlage für das Zustandekommen einer entzündlichen Verklebung mit dem Ovarium gegeben, die allmalig zur festen Verwachsung wird. Kommt nun gerade an diese Verwachsungsstelle ein atretischer hydropischer Follikel zu liegen, so wird die Seheidewand zwischen ihm und dem Tubensack durch Druckatrophie zu Grunde gehen können. Jetzt haben wir eine wirkliche Tuboovarialcyste: 
Sowohl Tuben- wie Ovarialgewebe sind in ihrer Wand nachzuweisen. Zuweilen findet man auch innen noch eine ringförmige oder halbmondähnliche Falte, welche auf die frühere Scheidewand mehr oder minder deutlich schliessen lässt, während aussen vielleicht eine ringförmige Einschnürung die ehemalige Verschmelzungsstelle bezeichnet; in anderen älteren Fällen wird der Uebergang makroskopisch überhaupt nicht mehr nachweisbar sein. - Die Ovarialcyste braucht aber nicht immer von einem Hydrops folliculi herzurühren, sondern dieselbe Infection, welche den perisalpingitischen Process hervorruft, kann auch eine Follikelcyste ${ }^{1}$ ) des Eierstocks zur Folge haben; oder gelegentlich betheiligen sich auch Corpusluteumcysten ${ }^{2}$ ) an der Bildung der Tuboovarialcysten.

Der einzige Gegner dieser Anschauungen ist Zahn ${ }^{3}$ ), welcher trotz der Beobachtungen der oben genannten Autoren sagt: "Niemand hat bis jetzt einen Tubarhydrops beobachtet und mitgetheilt" (vergl. ${ }^{2}$ ) u. ${ }^{3}$ ), „bei dem die Fimbrien in der Weise ins Tubeninnere eingestülpt gewesen waren, wie dies Burnier als etwas ganz gewöhnlich und nothwendig Vorkommendes anzunehmen geneigt ist".

Hierzu bemerkt Orthmann ${ }^{4}$ ) mit Recht:

"Ein derartiger Befund ist allerdings, wenn auch nicht etwas Nothwendiges, so doch etwas ganz Gewöhnliches, wie das jeder bestätigen kann, der häufiger Gelegenheit hat, Adnex-Operationen zu machen und hierbei die betreffenden erkrankten Organe zu besichtigen".

Wir düren daher diese Entstehungsart der Tuboovarialcysten als $z$ weifellos sicher bewiesen annehmen. Dass Aussehen eines solchen Adnextumors ist, wie auch überall hervorgehoben wird, von typischer Retortengestalt. Den Hals bildet die Tube, im uterinen Theil meist wenig verdickt, abdominalwärts in der Regel cylindrisch aufgetrieben, während der kugelförmige Bauch der Retorte von der Ovarialcyste geliefert wird. Wir haben also hier (ich betone das im Hinblick auf eine weiter unten zu beschreibende, ganz andere Morphogenese) in der Cystenwand sofort anschliessend an die letzten Tuben-, bezw. Fimbrien-

1) Burnier, Ueber Tuboovarialcysten. Zeitschr. f. Geb. u. Gyn. Bd. 5.

2) Rokitansky, Ueber Abnormitäten des Corpus luteum. 1859. Allg. Wiener med. Ztg. IV. Jg. No. 34 and 35.

3) Zahn, F. W., Ueber Tuboovarjalcysten. Virch. Areh. 151. S. 271.

4) Orthmann, Ueber d. Entstehungsweise d. Sactosalp. u. Tuboovarialcysten. Virchow's Arch. 155. S. 224. 
reste Ovarialgewebe, beide bisweilen, wie schon erwähnt, durch eine halbmondförmige Falte getrennt.

Vergegenwärtigen wir uns noch einmal obige Entstehung der Tuboovarialcyste: Die ehemalige Scheidewand wurde von 2 Schichten gebildet, vom Sactosalpinx einerseits, der Ovarialcyste andererseits, ganz gleichgültig, ob man sich diese aus einem primären Ovarialkystom oder einer Corp.-lut.-Cyste entstanden denkt. Die Fimbrien waren eingekrempelt und bildeten mit ihrer peritonealen Fläche zugleich den tubaren Antheil der Scheidenwand. Jetzt geht diese Scheidenwand durch Druckatrophie zu Grunde; die Communication zwischen Tubensack und Ovarialcyste ist fertig. Was findet man nun noch von den Fimbrien übrig? - Selbstverständlich sind sic, soweit sie die tubare Schicht der Scheidewand mitbildeten, ebenso wie die an der Scheidewand betheiligte, entsprechende Schicht der Ovarialcyste, vollständig zu. Grunde gegangen.

Soweit wäre die Morphogenese der Tuboovarialcysten als einfach zu bezeichnen. Nun giebt es aber, wie schon oben erwähnt, Fälle, bei denen sich die Fimbrien schön kranzförmig ausgebreitet an der Innenwand der Ovarialcyste angewachsen finden oder gar noch frei im Innern der Ovarialcyste flottiren! Diese Fälle lassen sich meines Erachtens durch die vorerwähnte Anschauung nicht erklären, obwohl dies von manchen Autoren versucht wird.

Nehmen wir zunächst die Burnier'sche Theorie, welche den meisten Anklang gefunden hat. Burnier nahm, wie schon erwähnt, mit Klob, Klebs, Virchow und anderen den abdominalen Tubenverschluss durch Verwachsung der peritonealen Fimbrienflächen an, genau ebenso, wie oben geschildert, den daraus resultirenden Pyosalpinx. Auch er liess in dem mit diesem verwachsenen Ovarium einen Follikel hydropisch werden und die Scheidewand zwischen diesem und dem Tubensack zu Grunde gehen. Nun aber sollte, um den Fimbrienbefund im Inneren der Ovarialcyste zu erklären, blos die Ovarialschicht der Scheidenwand atrophiren, die tubare Schicht der verklebten Fimbrien aber nur derartig gedehnt. werden, dass die Fimbrien an ihren Verwachsungsstellen sich wieder trennten, sich einzeln oder in kleineren oder grösseren Strähnen separirten, und nun sollten sie, da ja keine Wand sie mehr hinderte, sich in die Ovarialcystenhöhle umschlagen und dort frei flottiren! Jetzt hätten sie freilich auch an der Ovarialcysten- 
wand anwachsen können, und man hätte den oft erhobenen, oben beschriebenen Befund erklärt!

Aber man fragt sich doch, warum ist denn blos die eine Schichte der Scheidenwand, die vom Ovarium gelieferte, atrophirt? Warum nicht auch die Fimbrien, soweit sie an der Scheidewand betheiligt waren? Ist es überhaupt denkbar oder erwiesen, dass die Fimbrien gerade an ihren früheren Verwachsungsstellen sich wieder trennten? Und dann müsste die Trennung mit mathematischer Genauigkeit gerade am Verwachsungspol erfolgen, um die schönen ausgebreiteten Fimbrien wieder zum Vorschein kommen zu lassen! Waldstein ${ }^{1}$ ) macht hierüber die Bemerkung: „Sobald aber die Fimbrien eingerollt und untereinander verklebt sind, habe ich nur ein einziges Mal -- und da dürfte der Beginn der Beginn der Erkrankung auf einige Monate zurückzudatiren sein - die eingerollten Fimbrien noch so gut erhalten gefunden, dass eine Wiederentfaltung derselben vorstellbar gewesen wäre".

Man sieht, dass die Burnier'sche Entstehungsweise, sobald man sie genau verfolgt, mindestens sehr unwahrscheinlich, wenn nicht unmöglich ist. Immerhin hat sie in jüngster Zeit noch in Orthmann ${ }^{2}$ ) einen Vertheidiger gefunden.

Eine andere Theorie zur Errklärung des Befundes, wo die Fimbrien frei in der Ovarialcyste flottiren oder sich an ihrer. Wand angewachsen finden, ist die sogenannte Richard'sche Ovulationstheorie, der sich Klob, von Rosthorn, Rokitansky, Gottschalk und andere anschlossen. Richard ${ }^{3}$ ) nimmt an, dass die Fimbrien zur Zeit der Ovulation das Ovarium umklammern. Der Follikel platzt; da er erkrankt ist, schliesst er sich nicht: ${ }_{n} \mathrm{La}$ scène change! la vésicule malade ${ }^{4}$ ) ne se referme pas; car au lieu de former un corps jaune, elle continue de verser dans la trompe le liquide morbide qui la distend. C'est désormais uu kyste de l'ovaire, et ainsi croissent ensemble, d'une côté, la tumeur ova-

1) Waldstein, Ueber periphere Tubensäcke etc. Mon. für Geb. und Gyn. 1900. Bd. 11. S. 416.

2) Orthmann, Virchow's Archiv. 155.

3) Richard, A., Sur la communication des certains cystes de l'ovaire dans la trompe uterine (Cystes tubo-ovariens). Paris 1853 und Bulletin de l'academie de médecine. Bd. 22. S. 356 (eitirt nach Burnier).

4) Von einigen Autoren (Burnier, Waldstein u. A.) wird angenommen, Richard habe $\mathrm{n}$ ur an einen physiologischen Vorgang gedacht. Das ist falsch! Er spricht ausdrücklich von "malade", hat also sicher an pathologische Veränderungen, vielleicht an einen Katarrh gedacht. 
riennne, de l'autre la trompe incessament distendue par le liquide qu'elle reçoit.

Tel est le mode de formation des kystes tuboovariens".

Also während der Follikel weiter secernirt, verlöthet die Tube mit ihm. Letenneur nimmt nach Burnier auch an, dass die Fimbrienränder mit den zerrissenen Follikelrändern zusammenwachsen sollen. Auch von Rosthorn ${ }^{1}$ ) sagt: „Gelegentlich des Ovulationsprocesses schlüpft die vorher catarrhalisch erkrankte Tube in den geplatzten Follikel. Der Pavillon verwächst mit der Follikelwandung und es entwickelt sich eine Corp.-lut.-Cyste".

Orthmann ${ }^{2}$ ) bemerkt jedoch hierzu sehr richtig: "Erstens ist die bei dem Ovulationsprocess in dem sich eröffnenden Follikel entstehende Oeffnung so minimal, dass es meiner Ansicht nach ganz undenkbar ist, dass der ganze' Tubenpavillon in denselben hineinfallen oder hineinschlüpfen könnte; es wäre dies vielleicht für eine oder 2 Fransen möglich, aber das dürfte wohl kaum genügen, um einen vollständigen Verschluss herbeizuführen"..... "Zweitens müsste die Mehrzahl der Tuboovarialeysten, wenn die von Rosthorn'sche Ansicht richtig wäre, in Bezug auf ihren ovariellen Theil aus Corp. lut.-Cysten bestehen, was von Rosthorn auch selbst zugiebt; dies widerspricht aber den thatsächlichen Verhältnissen".

Ausserdem möchte ich fragen: Was hinderte wohl die Fimbrien daran, wieder herauszuschlüpfen, wirklich zugegeben, sie wären in den Follikel hineingeschlüpft? Ehe eine Verwachsung eintreten könnte, dürften Lageveränderungen der Patientin, Peristaltik der umgebenden Darmpartieen oder Anstauung des aus der Tube fliessenden Secrets die Fimbrien längst wieder hinausgeschafft haben. Gegen die "Ovulationstheorie" spricht aber vor allem, dass eine Umklammerung des Ovariums durch das Fimbrienende während des Ovulationsprocesses garnicht stattfindet, wie ja von der Mehrzahl der Forscher heute angenommen wird.

Giebt man jedoch das Umklammertwerden des Ovariums zu, dann müsste man doch vielmehr die Fimbrien an der Aussenseite der Ovarialcyste angewachsen finden, und da sie mit ihren epithelisirten Innenflächen hier nicht anwachsen konnten, könnte nur eine adhäsive Verklebung ihrer peritonealen Ränder am Ovarium

1) v. Rosthorn, Billroth-Festschrift. 1892. S. $12 \mathrm{sub} \mathrm{II}_{2}$.

2) Orthmann, Ueber die Entstehungsweisen etc. Virchow's Arch. 155. 
sie dort festgehalten haben. In der Literatur findet sich auch in der That ein solcher, allerdings vereinzelter Bericht von Blasius ${ }^{1}$ ): „Tubae ad novem vel decem pollicum longitudinem productae super ovaria quodammodo erant extensae et cum his fere ubique imprimis autem ad partem sinistriatam concretae, ita quidem, ut earum fines distincte cognosci possent, quorum sinistriae ad instar digitorum manus distentorum parti externae superficiei anterioris tumorum adjacebant". Es dürfte sich hier jedoch entweder um ein zufälliges Anlagern der Tube an das Ovarium handeln oder nach Orthmann ${ }^{2}$ ) "die Ausdehnung der Fimbrien auf der Aussenfläche des Ovariums ist erst secundär durch das Wachsthum des Ovarientumors zustande gekommen".

Verwandt mit dieser Richard'schen "Ovulationstheorie" ist die Veit'sche ${ }^{3}$ ) "Katarrhtheorie". Nachdem Veit nämlich von der Zweifelhaftigkeit einer Fimbrienannäherung an das Ovarium gesprochen, sagt er: „Es dünkt uns wahrscheinlicher, dass ein Katarrh der Tube und der Graal'schen Follikel den Ausgangspuntet bildet, dieser zur gleichzeitigen Anlöthung beider Organe Veranlassung giebt und hinterher erst die entstandenen Säcke in Communication treten". Auch diese Theorie kann den einen Befund nicht ererklären, bei dem die Fimbrien an der Cystenwand innen fächerförmig ausgebreitet angewachsen sind oder in der Cyste frei flottiren:

Gottschalk ${ }^{4}$ ) versuchte diesen Befund so zu erklären, dass die gesunde Tube mit ihrem Fimbrienende in eine geplatzte primäre Ovarialcyste hineinschlüpfe und dort anwachse. Wenn dieser Vorgang vielleicht nicht unmöglich ist, so hat er doch vor allem dieselben Gründe gegen sich, wie sie oben gegen das Hineinschlüpfen der Tube in einen geplatzten Ovarialfollikel geltend gemacht wurden. Ausserdem spricht die Häufigkeit des erwähnten Befundes und nicht am wenigsten die Thatsache dagegen, dass der der Tube zunächstliegende Abschnitt, der Cyste, wie wir zeigen werden, in vielen Fällen gar kein Ovarialgewebe ist.

1) Blasius, Commentatio de hydrope ovariorum profluente. Halae 1834. (Citirt nach Burnier.).

2) Orthmann, Virchow's Arch. 155. S. 229.

3) Veit, Krankheiten d. weiblichen Geschlechtsorgane. Erlangen 1867. S. 481 (citirt nach Burnier).

4) Gottschalk, Centralbl. für Gyn. 1891. No. 19, 22. 1898. No. 38. 
Ja in manchen Fällen, wie sie besonders $\mathrm{Zahn}^{1}$ ) und Waldstein $^{2}$ ) anführen, bildet das Ovarium nicht einmal einen wesentlichen Bestandttheil der Cystenwand, sondern findet sich noch.als selbstständiges Organ entweder in oder an derselben angelöthet (cfr. auch unsern Fall Katschker weiter unten Fig. 3) oder gar nicht mit der Cyste in Berührung [Zedel$\left.\left.{ }^{3}\right)\right]$. Zahn und Waldstein wenden dafür den Ausdruck "peripherer Tubensack" an. Besonders Zahn hat den sonst nur in wenigen Fällen beschriebenen Befund $15 \mathrm{mal}$ erheben können, dass das Ovarium nur an der Cystenwand an- oder in sie eingelagert ist, jedoch ${ }_{n}$ keinen wesentlichen Bestandtheil der Cystenwand bildet". - "Diese selbst", sagt er, "wird also fast durchweg von der Tubenwand gebildet und zwar in ihrem Schlauchtheil von der mehr oder weniger erweiterten Ampulle und in ihrem äusseren kugeligen Abschnitt von dem, wie wir später sehen werden, stark ausgedehnten Endtheil dieser und dem Infundibulum".

Zahn spricht sich aber weiterhin nirgends deutlich aus, ob er sich den Hydrosalpinx durch Verklebung der abdominalen Fimbrienffechen oder auf andere Weise entstanden denkt. Er scheint aber ein Zusammenwachsen der Fimbrien mit ibren Schleimhautflächen anzunehmen, eine Ansicht, die meines Wissens Niemand theilt. Wenigstens fasse ich seine Aeusserung so auf: "Burnier will nicht zugeben, dass die entzündeten Fimbrien, deren Epithel doch gewiss in Folge der Entzündung häufig theilweise oder auch ganz abgelöst ist, mit einander verwachsen können".

Gegenüber allen diesen erwähnten Erklärungsversuchen erscheint mir die Deutung, welche Professor Pfannenstiel 1899 in den Verhandlungen der deutschen Gesellschaft für Geburtshilfe und Gynaekologie (S. 218) gab, die einzig plausible zu sein. Pfannen'stiel hält nämlich den sogenannten "peripheren Tubensack" für eine "Pyocele peritubaria", eine Auffassung, die ich in dieser Arbeit ausführlicher begründen soll: Der "Tubensack" wird nur theilweise von der Tube gebildet. Die eigentliche Cyste, an deren Innenwand die Fimbrien angewachsen sind, ist aber eine "Pyocele peritubaria“, welche sich um das infolge von Ausfliessen infectiösen Tubensecrets entstandene

1) Zahn, F. Wilh., Ueber Tuboovarialcysten. Virohow's Archiv. 151.

2) Waldstein, Ueber periphere Tubensäcke etc. Mon. für Geb. und Gyn. 1900 . Bd. XI. S. 412.

3) Zedel, Ztschr. für Geb. und Gyn. 1894. 
Exsudat als Abkapselungsmembran bildet, in ganz analoger Weise, wie sich um die Haematocele nach Extrauteringravidität eine solche Abkapselungsmembran bildet. (vergl. Fig. 2).

Figur 2.

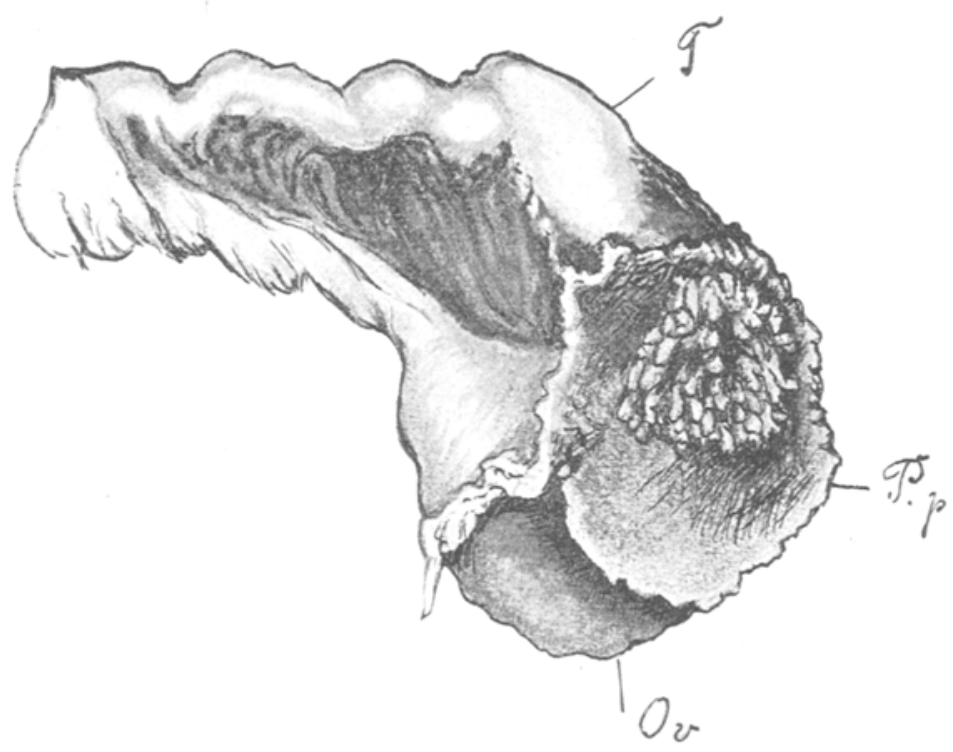

Frische "Pyocele peritubaria". Sie umgiebt das Tubenende, sodass die Fimbrien in der Pyocele frei flottirten. Sie war mit dem Ovarium und dem Rectum verlöthet. Gezeichnet ist nur der peritubare Pyocelentheil.

T. 'Tube. - Ov. Ovarium. - P. p. Pyocele peritubaria.

Da die Tube durchgängig geblieben war und mit ihrem schön ausgebreiteten Fimbrienende im Exsudat flottirte, wird sie dann nach Bildung der peritubaren Pyocelenwand allseitig fest von ihr umschlossen werden. Die Fimbrien flottiren jetzt entweder weiterhin frei im Exsudat im Cysteninnern oder können an der Innenwand der "Pyocele peritubaria" festwachsen. - Besteht nun gleichzeitig eine Ovarialcyste, sei es nun ein atretischer, cystisch dilatirter Follikel oder ein echtes Kystom, welches an die "Pyocele peritubaria" anstösst, so kann natürlich durch Druckatrophie der Wand zwischen Pyocelenhohlraum und Ovarialcyste eine Communication zwischen beiden eintreten. Dann wird der der Tube zunächstliegende Theil der Cystenwandung der "Tuboovarialcyste" von der ehemaligen "Pyocele peritubaria", der übrige von der Ovarialevste ge- 
bildet! Demgemäss können sich die Fimbrien, falls sie nicht in dem Cysteninneren frei flottiren, nur in dem der Tube zunächstliegenden Pyocelentheil der Cystenwand angewachsen finden!

Auch Zahn hat an der Wand des "Tubensacks", sich "oft bis weithin in die Hauptcyste" fortsetzend, sowohl makro-, wie mikroskopisch Fimbrienreste constatirt. Zahn unterscheidet in der "Tubensackwand" drei Schichten: "eine innere epitheltragende" (Fimbrienepithel? - Verf.), „aus mehr oder weniger zellreichem Bindegewebe bestehende; eine mittlere bindegewebe- und sehr gefässreiche, in welcher häufig in Bündeln beisammenliegende glatte Muskelfasern vorhanden sind, und eine äussere, gewöhnlich nur aus Bindegewebe bestehende, wenig gefässreiche Schicht. Verschiedenheiten von diesem Bau der Wand kommen nicht nur bei verschiedenen Cysten vor, - sondern auch an verschiedenen Wandtheilen derselben Cyste. So können z. B. die Muskelfasern der mittleren Wandschicht in grösserer oder auch in kleinerer Anzahl vorhanden sein, ja zuweilen sogar ganz fehlen, sodass diese Schicht nur aus ziemlich straffem Bindegewebe besteht, und es kann auch vorkommen, dass sowohl in der inneren Schicht, als auch im inneren Theil der äusseren Schicht vereinzelte Muskelfasern sich vorfinden."

Ich selbst habe nun in den untersuchten Peritubarpyocelenmembranen ähnliche Beobachtungen machen können: Die innerste, epitheltragende Schicht reicht allerdings nur so weit, wie der Fimbrienbelag reichte. Genau so weit blos waren hier auch einzelne glatte Muskelbündel zu verfolgen, deren Zusammenhang mit den Fimbrien offenkundig war. Von gleichen Befunden berichtet auch Waldstein in der oben erwähnten Arbeit, die ein Jahr nach der Publication der Pfannenstiel'schen Theorie erschien, und in der er die "peripheren Tubensäcke" auf alte Hämatocelen zurückführen will: "Fine Epithelauskleidung der Wand konnte nur an den von den Fimbrien und deren Epithelüberzug bedeckten Wandstellen gefunden werden. Die übrigen Wandpartien entbehrten eines Epithelüberzuges."

In der mittleren Schicht, die, welche nach Pfannenstiel gerade die um das Exsudat neugebildete Pyocelenmembran darstellt; habe ich nie Muskelgewebe gefunden. Sie besteht vielmehr durchweg aus Bindegewebe von lamellösem, concentrischem Bau verschiedenen Alters, ist kleinzellig infiltrirt und zeigt nach dem Cysteninnern zu an den Stellen, wo der Fimbrienbelag bereits aufgehört hat, nur epithelloses Granulationsgewebe. Zahn sagt ja 
selbst, dass auch bei seinen Beobachtungen oft die mittlere Schicht ohne Muskelfasern gewesen sei und nur aus " straffem Bindegewebe " bestanden habe. Ich bin deshalb geneigt, die Stellen, an denen er in der mittleren Schicht glatte Muskelfasern gefunden, seiner dritten äusseren Schicht hinzuzurechnen; diese ganze dritte Schicht dürfte aber aus Ligamentresten bestanden haben, zu denen auch die fraglichen glatten Muskelfasern zu rechnen sind. Denn die meisten dieser Tumoren liegen pseudointraligamentär. Auch Waldstein sagt: "Weiterhin muss bervorgehoben werden, dass die peripheren Tubensäcke insgesammt an der hinteren Fläche des Lig. latum, tief unten im Douglas gelegen waren."

Der Inhalt der eröffneten Cysten ist manchmal eitrig, manchmal eitrig-serös oder auch rein serös, was aber nicht ausschliesst, dass er früher rein eitrig gewesen ist. Nie habe ich jedoch im Cysteninhalt Blut oder dessen Residuen nachweisen können. Hierauf komme ich indessen noch zurück.

Einer der Pfannenstiel'schen Auffassung näherstehenden Anschauung giebt Zedel ${ }^{1}$ ) Ausdruck, wenn er den „peripheren Tubensark" für eine durch Peritonitis entstandene "Adbäsionseyste" anspricht. Er hat einen ganz analogen Befund, wie ihn der Fall Katschker weiter unten bietet, erheben können, geht aber von der wohl schwer verständlichen Anschauung aus, dass peritoneale Verwachsungen und Pseudomembranen durch Druck aneinandergepresst werden und mit einander verkleben können. Dieser Vorgang ist nirgends beobachtet und an sich unwahrscheinlich, wie weiter unten gezeigt werden soll. Zedel sagt: „Die Art der Entstehung ist dann so zu denken, dass sich zahlreiche peritoneale Adhäsionen um die Ampulle des Eileiters bilden, die unter einander verkleben und so einen das Ostium abdominale umgebenden Hohlraum einschliessen. Letzterer wird durch das Secret der entzündeten Tube immer mehr zu einer prall gefüllten Cyste ausgedehnt, in welche das Fimbrienende frej hineinragt. Die Fimbrien der nur mässig erkrankten Tube brauchen hierbei durchaus keine bemerkenswerthen Veränderungen zu erleiden, mit der Wand zu verkleben, sich wie bei beginnendem Tubenverschluss einzuscblagen oder gar unter einander zu verwachsen. Die entzündliche Wucherung des Bindegewebes bedingt ferner eine bedeutende Verbreiterung der Cystenwand. Weiterhin schreiten die Entzündungsprocesse zu einer starken

1) Zedel, Ztschr. für Geb. und Gyn. 1894. 
eitrigen Gewebsinfiltration, namentlich in den inneren Schichten mit nachheriger Einschmelzung des Gewebes fort. Dass die Innenwand unserer Cyste zum Theil von Granulationsgewebe gebildet wird, ist unter den gegebenen Verhältnissen ebensowenig auffallend, wie in einem gewöhnlichen Abscess. - Gerade aber die Annahme der letzten Behauptung dürfte keine allgemein richtige sein: Wenn Pseudomembranen sich aneinanderlegen und selbst mit einander zu einer relativ dicken Wand verwachsen könnten, so würden sie doch dem sie entfaltenden und anspannenden Eiter eine glatte Fläche entgegenbringen, und am allerwenigsten dürfte man hier an diesen mechanisch gedehnten Innenschichten deshalb Granulationsgewebe zu erwarten haben, noch dazu, wo eine "Pseudomembran" und eine "Adhäsion" selbst das Product einer Entzündung sind und aus straffem, narbigem Bindegewebe bestehen, das einer „Einschmelzung des Gewebes", wie sie Zedel annimmt, recht energischen Widerstand entgegensetzen würde. Auch könnte, selbst wenn viele "Pseudomembranen" sich aneinanderlegten und verwüchsen, doch schwerlich eine allseitig $3-10 \mathrm{~mm}$ starke Cystenwand resultiren, wenn dies Z edel aueh zu erklären sucht: "Die entzündliche Wucherung des Bindegewebes bedingt ferner eine bedeutende Verbreiterung der Cystenwand." Alle diese Erwägungen lassen den Schluss nicht allzu fern erscheinen, dass man in der Zedel'schen "Adhäsionscyste" vielmehr eine Pfannenstiel'sche "Pyocele peritubaria" zu erblicken hat. Hierfür spricht aber noch Folgendes, was Zedel weiterhin sagt: "Einige Male senkt sich dicht neben dem Fimbrienende das Epithel ähnlich einer tubulösen Drüse tief in die Cystenwand hinein" ... "Ausserdem sieht man in der Cystenwand, besonders innerhafb des kleinzellig infittrirten Gewebes zahlreiche, mit Cylinderepithel ausgekleidete, längliche Hohlräume, welche gewöhnlich zu mehreren bei einander liegen. Thre Längsachse verläuft meist ziemlich senkrecht zur Cystenwand. Centralwärts verliert sich die epitheliale Auskleidung der Räume nicht selten in der kleinzelligen Infiltration. Am zahlreichsten sind diese Epithelräume in der Nähe der einmündenden Tube; in den distalen Theilen der Cyste, deren Gewebe durch die starke Dehnung der Wand mehr weniger atrophirt ist, fehlen dieselben ganz."

Wäre nun die Zedel'sche Anschauung, dass sich die „Adhäsionscyste" aus unter einander verwachsenen Pseudomembranen gebildet habe, richtig, so wäre eine Einsenkung von einigen Fim- 
brien „ähnlich einer tubulösen Drüse" in die Cystenwand oder gar die Bildung von mit Cylinderepithel ausgekleideten Räumen, die noch dazu mit ihren Längsachsen zur Cystenwand senkrecht stehen, unmöglich. Fasst man dagegen die Wand der Cyste als eine Pfannenstiel'sche Pyocelenmembran anf, so kann man sich sehr gut und ungezwungen vorstellen, dass einzelne Fimbrien sich bei der Bildung der Pyocele in deren Wand einlagerten und so später die obigen Bilder darboten. Das ist wohl möglich, wenn sich um das Exsudat herum eine granulirende Schwarte bildet, nicht aber, wenn abschliessende adhäsive Pseudomembranen vow Eiter gedehnt werden und so den anliegenden Fimbrien doch nur eine meist prall gespannte, glatte Fläche zukehrten. Alle diese Erwägungen lassen die Zedel'sche Anschauung als sehr unwahrscheinlich erscheinen.

Derartige mit Fimbrienepithel ausgekleidete Hohlräume habe auch ich gesehen, ebenso Waldstein, der dic der Tubenmündung zunächst liegenden von den Fimbrien ableitet, bei den weiter distal gelegenen aber eine Abstammung von mit Cylinderepithel versehenen Schläuchen des Par- und Epoophoron als möglich in Betracht zieht. Ich halte die der Tubenmündung zunächst liegenden für Fimbrienderivate, die distal gelegenen für atretische Follikel.

Ich komme jetzt zur ausführlicheren Besprechung der jüngsten Arbeit über Tuboovarialcysten, der Arbeit von Waldstein ${ }^{1}$ ). W. will diejenigen Tuboovarialcysten, bei denen sich die Fimbrien im Cysteninnern frei flottirend oder an der Cystenwand angewachsen finden, dadurch erklären, dass er sie genetisch auf eine frühere Extrauteringravidität mit Hämatocele zurïckführt. Er sagt u. a.: "Ich sehe die catarrhalische Erkrankung des Uterus und der Tuben als die erste Ursache für die Entstehung peripherer Tubensäcke an, deducire aus derselben jedoch nicht unmittelbar die Pelveoperitonitis mit resultirender Schwielenbildung um die Tube; jch fasse vielmebr den Uterus- und Tubencatarrh als das eine Tubargravidität provocirende Moment auf. Durch diese kommt es zur Haematocelenbildung und durch Organisation der Hämatocele zur Entwicklung eines peripheren Tubensackes. Auf diese Weise glaube ich die entzündlichen Veränderungen am Genitale hinlänglich gewürdigt zu haben und den Zusammenhang zwischen Tubenkatarrh und peripherer Schwielenbildung in logischer Weise erbracht zu haben."

1) Waldstein, Ueber periphere Tubensäcke und ihre Bedeutung für die Aetiologie der Tuboovarialcysten. Mon. für Geb. und Gyn. Bd. XI. 1900. 
An anderer Stelle fährt W. dann fort: „Auf einen weiteren Punkt will ich noch hinweisen. Es ist dies die Doppelseitigkeit der peripheren Tubensäcke, wie wir sie mit Wahrscheinlichkeit im Fall IV annehmen müssen. Wenn auch doppelseitige Extrauteringraviditäten nicht häufig zur Beobachtung gelangen, so sind derartige Fälle doch hinlänglich oft bekannt geworden, ich verweise nur auf die jüngst gemachte Demonstration eines einschlägigen Falles in der Section für Gynäkologie des College of Physiciains in Philadelphia durch Dorland, der weiterer 15 analoger, aus der Literatur gesammelter Fälle Erwähnung that. In der Doppelseitigkeit, wie ich sie in einem Falle peripherer Tubensäcke gefunden haben dürfte, sehe ich somit kein Argument, das ihrer Ableitung entgegengestellt werden könnte."

Gegen die Ableitung der "peripheren Tubensäcke" von Hämatocelen möchte ich aber doch gerade die häufige Doppelseitigkeit anführen, die mit der Seltenbeit der doppelseitigen Extrauteringravidität nicht im Verhältniss steht. Wenn auch der Gegenbeweis gegen die Entstehung des peripheren Tubensackes via Hämatocele sich bei unserer heutigen Kenntniss über derartige Adnextumoren schwer wird erbringen lassen und man von vornherein diese Entstehungsmöglichkeit zugeken wird (dafür spricht ebenso wenig), so dürfte sich doch der weitaus grösste Theil der obigen Befunde durch rein entzündliche Processe ohne das $Z$ wischenglied einer Extrauteringravidität mit Hämatocele erklären lassen, auf dem näheren Wege einer „Pyocele peritubaria", ganz abgesehen davon, dass noch weitere Gründe hierfür sprechen: So möchte ich nämlich ein Merkmal, das W. für die Herleitung von einer Hämatocele als beweisend ansieht, direct für die Entstehung nach Pfannenstiel heranziehen: den Umstand nämlich, dass die äusseren Schichten der lamellös gebauten Cystenwand aus "Bindegewebe älteren Datums" bestehen. "ES ist dies ein Verhalten," sagt W., das gerade für organisirte Hämatocelen von Mandl und Schmit als charakteristisch hingestellt wurde."

Dies möchte ich ebenso gut von der Pyocelenmembranbildung behaupten, wie dies die mikroskopische Untersuchung meiner Fälle deutlich erweist; denn auch bei der Bildung der "Pyocele peritubaria" dürfte ebenfalls die Organisation des Exsudats von aussen nach innen erfolgen. Hierfür spricht einmal die Mehrzeitigkeit der concentrischen Schichten, die äusseren weniger kernhaltig wie die 
inneren, dann aber vor Allem die oben bereits erwähnte Anwesenheit von frischem Granulationsgewebe an der Innenfläche der Cyste, wie dies ja auch von $\mathrm{Zahn}$ hervorgehoben wird. Als weiteres wichtiges Argument gegen die Ableitung von Hämatocelen möchte ich das Fehlen von Blut oder dessen Residuen im Cysteninhalt anführen. Waldstein sucht dies von vornherein zu entkräften; er sagt nämlich: „Erst kürzlich untersuchte ich eine Hämatocele von ca. sechswöchentlichem Bestande, die in ihrer Struktur, in der Art der Organisation ihrer Wand mit Wandstellen peripherer Tubensäcke die höchste Aehnlichkeit bot. Diese wurde noch dadurch gesteigert, dass nur erstaunlich geringe Mengen von Blutfarbstoff und Fibrin in ihr nachweisbar waren. Wir müssen uns vorstellen, dass die Organisation äusserst schnell stattfand, desgleichen, dass die Deportation des Blutfarbstoffs aus der Hämatocele (nach Sänger durch Leukocyten bedingt) eine äusserst energische war. So ist es zu erklären, dass ich in Wandstèllen der erwähnten und anderer Hämatocelen, ebenso wie in peripheren Tubensäcken oft nichts als lamellöses Bindegewebe finden konnte."

Wenn aber nun in der weitaus grössten Zahl der Fälle von ,peripheren Tubensäcken" der Nachweis von Blutfarbstoff völlig oder fast völlig negativ ausfällt, so ist dieses Ergebniss doch nicht so belanglos, wie W. es hinstellen möchte. Eine Spur von Blutpigment oder andere Zeichen einer früheren reichlichen Anwesenheit von Blut im Cysteninhalt müsste doch besonders in jüngeren Fällen irgendwo in der Wand noch nachweisbar sein, wenigstens mikroskopisch. Statt dessen nur seröser bis serös-eitriger Cysteninhalt und in der Wand nur Granulationsgewebe und Bindegewebe ohne Pigmentzellen!

Wenn ich also das gelegentliche Entstehen von "peripheren Tubensäcken" via Hämatocele nicht in Abrede stellen will, glaube ich aber doch aus den eben angeführten Gründen bewiesen zu haben, dass die meisten dieser Gebilde auf eine "Pyocele peritubaria" im Sinne Pfannenstiel's zurückzuführen sind.

Der späteren Forschung ist es vorbehalten, darüber zu entscheiden, welche von beiden Morphogenesen beim Zustandekommen dieser Art von Tuboovarialcysten in Betracht kommt, ob ein ,,peripherer Tubensack" via Hämatocele nach Waldstein oder eine Pfannenstiel'sche "Pyocele peritubaria" oder auch, wie wahrscheinlich, beide. Denn haben wir erst einen "peripheren Tuben- 
sack", so sind für das Zustandekommen einer Tuboovarialcyste die Vorbedingungen vorhanden, ganz genau so, wie wenn wir es mit einer „Pyocele peritubaria" zu thun haben. Der Vorgang spielt sich dann so ab, wie ihn Waldstein in seinen schematischen Zeichnungen zur Darstellung bringt. Das Ovarium kann, wie schon oben erwähnt, an der Wand der "Pyocele peritubaria" angelöthet sein (unser Fall Katschker und Zahn's Fälle), ein Follikel kann in der Scheidewand hydropisch werden (Fall III von Waldstein) oder eine Corpns-luteum-Cyste oder ein primäres Ovarialkystom (unser Fall Pantke) kann durch Druckatrophie der Scheidewand mit der "Pyocele peritubaria" in Communication treten; immer werden wir jedoch einen Vorgang haben, der als Endresultat den Befund zeigt, dass die Fimbrien in der Tuboovarialcyste frei flottiren oder secundär an deren Wand angewachsen sind. - Die Scheidewand zwischen ursprünglichem ,peripheren Tubensack" und Ovarialcyste ist entweder schon durchgängig (Fall VI von Waldstein, unser Fall Schaal), aber noch erhalten; oder aber auch sie jst nur noch in einer aussen verlaufenden Schnürfurche oder einer halbmondförmigen Falte im Innern oder überhaupt nicht mehr zu erkennen (unser Fall Hoffmann). Diese Entstehung ist also gegensätzlich verschieden von der Anfangs dieser Arbeit erwähnten Morphogenese via Sactosalpinx (unser Fall Cohn), bei der sich niemals die Fimbrien an der Innenfläche der Tuboovarialcyste ausgebreitet flottirend oder angewachsen finden können.

Um nun noch zwei Folgezustände nicht unerwähnt zu lassen, die möglicher Weise bei derartig zu Stande gekommenen Tuboovarialcysten eintreten können, so erinnere ich zunächst an den unter dem klinischen Bilde des "Hydrops ovarii profluens" bekannten Zustand. Nassauer ${ }^{1}$ ) hat mehrere derartige Fälle veröffentlicht. Durch Exacerbationen eines Tubenkatarrhs kann in der von einer früheren Erkrankung herrührenden Tuboovarialcyste (Pyocele peritubaria) der Druck der Flüssigkeit ein so hoher werden, dass er den Widerstand der infolge der Entzündung stark geschlängelten Tubenwindungen überwindet und die Flüssigkeit infolge dessen nach dem Uterus zu abfliesst. Wiederholt sich das bei späterem Wiederansteigen des Druckes in der Cyste, so haben wir die "Hydrorrhoea ovarialis intermittens" Nassauer's.

1) Nassauer, Hydrorrhoea ovarialis intermittens. Zur Lehre von den Tub.-Ov.-Cysten. Münch. med. Wochenschr. 1900. No. 7-9. 
Ein anderer gelegentlich bei Tuboovarialcysten anzutreffender Befund ist nach $\mathrm{Zahn}^{1}$ ) das Vorhandensein von noch ovulationsfähigem Eierstocksgewebe in der Cystenwand. Z. meint hierüber: „Wenn man sich den oben geschilderten Bau der Tuboovarialcyste vergegenwärtigt, so erscheint es ganz selbstverständlich, dass innerhalb einer solchen Cyste die Befruchtung des Eies mit nachfolgender Weiterentwicklung desselben bis zur Reife des Fötus stattfinden kann. So lange in dem einen Theilbestandtheil der Cystenwand ausmachenden Eierstock noch Ovulation vorhanden ist, so lange können auch noch reife Eier in den Cystensack gelangen. Andererseits kann dies aber auch nach der Begattung für die Spermatozoën der Fall sein, da ja die Tube in der Regel durehgängig und manchmal sogar auf Seiten ihres uterinen Endes erweitert ist. Die im Cystensack vorhandene Flüssigkeit aber ist in den meisten Fällen für das Weiterleben des Eies und der Spermatozoën cbenfalls von günstiger Beschaffenheit. Sind nun beide Theile gleichzeitig hier vorhanden und findet eine Vereinigung zwischen ihnen statt, so steht der Anheftung und Weiterentwicklung des Eies nichts mehr im Wege. Die anatomischen Bedingungen hierfür und für die vollkommene Ausreifung der Frucht sind sogar die denkbar günstigsten. Ich halte es für sehr wohl möglich, ja sogar für sehr wahrscheinlich, dass ein grosser Theil der Extrauterinschwangerschaften, bei denen die Föten bis nahe oder auch ganz bis zur Reife gediehen, den Tuboovarialschwangerschaften angehören dürften."

Zahn führt dann noch einige einschlägige Fälle aus der Literatur an.

Ich lasse jetzt eine Reihe von Fällen folgen, wobei ich bemerke, dass ich hier nicht alle unsere Beobachtungen von Tuboovarialcysten bringe, sondern nur die ausgewählt babe, die eine stufenweise Entwicklung der Tuboovarialcyste klarlegen sollen.

Bei Besprechung der Entstehungsweisen der Tuboovarialcysten erwähnte ich zuerst als sicher bewiesene die, welche die Bildung eines Hydro- oder Pyosalpinx als Zwischenglied hat. Dieser entsteht primär durch Verwachsen der eingekrempelten Fimbrien, wie oben ausführlicher dargethan wurde; ein Follikel des mit ihm verwachsenen Ovariums wird am Platzen verhindert und giebt den Anlass zu einer Ovarialcystenbilduug, die dann nach Atrophie der

1) F. W. Zahn, Ueber Tuboorarialcysten. Virch. Arch. 151. 
Zwischenwand mit dem Sactosalpinx in Communication tritt, so dass wir dann schliesslich eine echte Tuboovarialcyste vor uns haben. Oder die Ovarialcyste braucht ihrerseits auch nicht von einem hydropisch gewordenen Follikel abzustammen, sondern kann eine Corpus-luteum-Cyste oder ein primäres Ovarialkystom sein.

Einen Befund, den wir als Vorstufe einer so entstandenen Tuboovarialcyste ansprechen müssen, habe ich in folgendem Fall erblicken dürfen:

Fall Cohn. Die 40 jährige Patientin ist verheirathet, regelmässig alle vier Wochen nicht besonders stark menstruirt; letzte Menstruation vor zwei Wochen. Vor 17 Jahren eine Entbindung; im selben Jalure ein dreimonatlicher Abort, dem nach einem Jahre ein solcher von serhs Wochen folgt. Vor Jahren machte sie eine heftige Unterleibsentzündung durch. Seit 1895 ist sie in Behandlung des Herrn Professor Pfannenstiel, der einen faustgrossen Tumor in der linken Beckenhälfte constatirte. Die entzündliche, schmerzhafte Geschwulst nahm zuerst $a b$, seit einem Jahre aber wieder zu, desgleichen auch im Allgemeinbefinden Schlaffheit und Müdigkeit. Schmerzen waren fast gar nicht vorhanden. Jedoch regte der Gedanke, es könnte später eine Operation unter schwierigeren Verbältnissen unumgänglich sein, die hochgradig nervöse Patientin so anf, dass auf ihr Drängen zur Operation geschritten wird.

Status: Mittelgrosse, leidlich genährte Person mit ängstlichem. Gesichtsausdruck und linksseitiger Facialisparese in allen Aesten. Geringe Beweglichkeit schon wieder vorhandeu. In der linken Beckenseite, über dasselbe hinausragend, ein ca. mannsfaustgrosser Tumor, leicht compressibel, macht einen cystenähnlichen Eindruck. Die rechten Adnexe gering vergrössert, jedoch ebenso wie die die linken bei Berührung sehr schmerzhaft.

Am 13. 2. 1900 wird in Chloroformäthernarcose die Laparotomie vorgenommen. Nachdem die Bauchböhle mittels eines suprasymphysären Fascienquerschnittes (Pfannenstiel) eröffnet ist, zeigen sich überall viele alte Adhäsionen und Pseudomembranen. Die rechten Adnexe werden zunächst in Angriff genommen. Das rechte Ovarium erweist sich als gesund; die rechte Tube als kolbig aufgetrieben und geschlängelt. Sie wird entfernt. Die linken Adnexe sind zu einem klein apfelgrossen Tumor verändert, der an der linken Tube hängt. Die Auslösung desselben ist sehr schwierig, gelingt aber theils stumpf, theils mit der Scheere, ohne dass die Cyste einreisst. Entfernung mit der Tubenecke. Schluss der Wunde und Bauchhöhle. Prima intentio. Am 11. 3. 1900 wird Patientin geheilt entlassen.

Pathologischer Befund: Die linke Tube ist uterinwärts auf etwa zwei Drittel ihrer Länge von normaler Dieke, im Ganzen $11 \mathrm{~cm}$ lang und mit Adhäsionsresten bedeckt. Abdominalwärts verdickt sie sich allmälig zu einem $\mathbf{2}$ cm langen, fingergliedstarken Kolben, der fest an eine kleinapfelgrosse Cyste angeschlossen ist. Ein durch das Tubenende und die Cyste gelegter, beide halbirender Schnitt lässt dann Folgendes sichtbar werden: Die Tube ist in ihrem abdominalen Theil hydropisch erweitert, die Fimbrien eingekrempelt und mit ihren abdominalen Flächen an einander gewachsen. An der Stelle 
ihres früheren Abdominalostiums ist die Tube mit der faustgrossen Cyste fest in der Ausdehnung eines Zehnpfennigstückes verwachsen. Hier ist die Wand der Cyste ca. 5-6 mm dick, während sie sonst nur eine Dicke von $2-3 \mathrm{~mm}$ hat. Hier sieht man ferner einige kleinere, stecknadelkopf- bis linsengrosse Cystchen in der Wand, welche sonst überall deutlich eine hellere, dem Cystenlumen zugekehrte Schichte und eine derbere, dunklere äussere Schichte zeigt. In die innere Schichte eingelagert und diese in zwei Blätter spaltend, ist an mehreren Stellen eine käsig-bröcklige, gelbe Masse, kalottenförmig der Cyste aufsitzend, eingebettet. Diese Masse wölbt das innere Blatt gegen das Cystenlumen mehrfach in einer Ausdehnung bis zu Zweimarkstückgrösse vor. Die Innenwand der Cyste ist spiegelnd glatt. Die mikroskopische Untersuchung ergiebt, dass die Wand der apfelgrossen Cyste grade in der Umgebung der Verwachsungsfläche mit dem Hydrosalpinx, wo sie 5 bis $6 \mathrm{~mm}$ dick ist, Ovarialreste führt. Die kleinen, hier gelegenen Cystchen sind Follikelcystchen. Sie tragen ebenso wie die grosse Cyste einschichtiges Cylinderepithel. Die Räume der Cystenwand, in denen sich die käsigen Massen befinden, sind von gleicher Auskleidung. Die rechte Tube bildet einen Hyprosalpinx; die Fimbrien sind eingekrempelt, mit ihren abdominalen Flächen so verwachsen, dass abdominalwärts ein voller Verschluss zu Stande gekommen ist. Durch eine klare, seröse Flüssigkeit war der abdominale Theil der Tube bis auf Daumenglieddicke aufgetrieben.

Wir haben es also hier links mit einer Ovarialcyste zu thun, welche mit einem Sactosalpinx zusammenhängt. Hier hätte durch Druckatrophie nur noch die trennende Zwischenwand zu Grunde gehen müssen, und wir hätten dann eine Tuboovarialcyste gehabt und zwar den Entstehungsmodus, den, wie oben erwähnt, frühere Autoren ebenfalls als sicher vorkommend angenommen haben. Der rechte Sactosalpinx ist als noch frühere Vorstufe als der linksseitige anzusprechen. Hier berühren sich Hydrosalpinx und Ovarium noch nicht; anch ist das Ovarium noch unverändert.

Eine weitere Stufe bildet der folgende Fall, in dem die Communication zwischen Tube und Ovarialcyste bereits eingetreten ist.

Fall Pantke. Pat. ist eine 42jährige, verheirathete Frau, regelmässig menstruirt bis vor 2 Jahren $4-5$ Tage lang. Seitdem traten manchmal Jängere Pausen ein, bis sich seit $1 / 2 \mathrm{Jahr}$ unregelmässige Blutungen einstellten. Vor 16 Jahren machte sie eine Bauchfell-, Blinddarm- und Gebärmutterentzündung durch, nach 2 Jahren eine weitere Unterleibsentzündung. Seitdem blieben dauernd Schmerzen in der rechten Seite, bis sich vor ca. 1 Jahre auch solche in der linken Seite hinzugesellen, die sich derartig steigern, dass P. um die Operation bittet. Die innere Untersuchung ergiebt, dass sich der Uterus in anteflectirter Stellung befindet. Portio für die Kuppe des Fingers durchgängig; links und hinten vom Uterus ein kindskopfgrosser, prallelastischer Tumor. Eine Probepunction ergiebt einige Epithelien, Leukocyten und Streptokokken.

Am 23. 10. 1896 Laparotomie. Der Uterus ist stark metritisch 
vergrössert und mit den Nachbarorganen fest verwachsen. Die Adhäsionen werden theils stumpf, theils mit der Scheere getrennt. Jetzt wird ein nahe der linken Tubenecke gelegener, anscheinend rom linken Ovarium ausgehender, retroligamentär entwickelter kindskopfgrosser Tumor erst sichtbar, der nur mit grosser Mühe hervorgewälzt werden kann. Hierbei reisst er ein und entleert aus 2 Stellen eine ziemliche Menge Eiters. Es gelingt durch sofortige Tamponade die Därme zu schützen. Darauf wird der Tumor, der an der Tube hängt, mit dieser und der zugehörigen Tubenecke entfernt. - Die rechten Adnexe sind mit Adhäsionen bedeckt; jedoch frei von Eiter und nicht vergrössert; sie bleiben darin. - Die Heilung nimmt wegen Vereiterung mehrerer Stichcanäle einen langsamen Verlauf; jedoch wird P. Ende Januar 1897 als geheilt entlassen.

Pathologischer Befund: Die linke Tube ist ca. $11 \mathrm{~cm}$ lang und verdickt sich abdominalwärts zum Theil bis zu einem Umfange von $7 \mathrm{~cm}$. Ihre Wände sind zum Theil bis $1 \mathrm{~cm}$ dick. Die Tube geht in eine kindskopfgrosse Cyste über. Nachdem diese aufgeschnitten, zeigt sich, dass die Tube in sie einmündet, wie ein Retortenhals in einen Retortenbauch, nur dass hier die Einmündung unter einem spitzen Winkel erfolgt. Von Fimbrien ist makroskopisch nichts zu sehen. Das Tubenlumen ist durchgängig. Die Cystenwand zeigt innen ein körniges Aussehen von gelber Farbe. Zwischen der Vorderfläche der Cyste und dem bei der Operation theilweise mitentfernten Lig. lat. spannt sich eine bei der Operation eingerissene Abscessmembran aus, die vielleicht $30 \mathrm{ccm}$ Eiter enthalten haben konnte. Zwischen diesem Raum und der grossen Cyste besteht keine Communication. Bei der mikroskopischen Untersuchung zeigt sich, dass dieser Raum von einer typischen $\mathrm{Ab}$ scessmembran überdacht wird, während das Lig. lat. seine vordere, die kindskopfgrosse Cyste seine hintere Begrenzung bildet. Diese selbst zeigt in ihrer Wand besonders in den der Tubenöffnung näher liegenden Partien fibrös entartetes Ovarialgewebe, während die Cyste selbst nach ihrem Epithelbau als Ovarialcystom angesprochen werden muss. Fimbrien sind auch mikroskopisch innerhalb der Cystenwand nicht mehr nachzuweisen, sondern es findet sich sofort neben dem deutlich erkennbaren Tubengewebe fibrös entartetes Ovarialgewebe an der Einmündungsstelle der Tube in die Cyste, und zwar so, dass sich an der Cystenwand überhaupt kein Tubengewebe findet.

Wir haben hier also in der kindskopfgrossen Cyste ein Ovarialkystom, während die Tube ursprünglich einen Sactosalpinx gebildet haben muss. Ovarialkystom und Sactosalpinx berührten sich, die Zwischenwand ging durch Druckatrophie zu Grunde, und so ist es möglich, dass wir an der Einmündungsstelle der Tube sofort neben ihrem Gewebe auch Ovarialgewebe finden. Hier liegt also eine echte Tuboovarialcyste mit dem Eingangs dieser Arbeit erwähnten Entstehungsmodus via Sactosalpinx vor.

Die folgenden 3 Fälle sollen nun die verschiedenen Entwickelungsstufen einer echten Tuboovarialcyste via "Pyocele peritubaria" Pfannenstiel klarlegen, der Form 
der Entstehung, die nach den Erörterungen dieser Arbeit nächst der eben beschriebenen wohl die häufigste sein dürfte.

Fall Katschker. Patientin ist eine 28jährige, verheirathete Frau, hat 4 mal geboren, 4 mal abortirt. Letzte Geburt vor einem Jahr, letzter Abort vor $3 / 4$ Jahren im 2. Monat. Schon seit der ersten Fellgeburt, die auf die erste Geburt folgte, Beschwerden: Leib- und Kreuzschmerzen, Ausfluss. Periode früher regelmässig; seit dem letzten Abort stärker mit unregelmässigen Blutungen, deren letzte vor 3 Wochen begann und 14 Tage dauerte.

Status: Kleine, normal gebaute Frau von leidlichem Ernährungszustand und etwas anämischem Aussehen. Innere combinirte Untersuchung ergiebt: Doppelseitiger Cervixriss, Uterus anteflectirt, nicht vergrössert; rechte Adnexe etwa apfelgross und schmerzhaft.

$\mathrm{Am}$ 9. 3. 1899 in Chloroformåthernarcose Laparotomie: Suprasymphysärer Fascienquerschnitt (Pfannenstiel). Der Uterus und die Adnexe erweisen sich als stark verwachsen und mit Adhäsionen bedeckt, die sich aber nicht allzu schwer lösen lassen. Es wird zunächst ein Theil des linken Ovariums, die linke Tube mit der Uterustubenecke entfernt, darauf das Uteruscavum an dieser Stelle ausgeglüht und vernäht. Die rechten Adnexe sind in einen apfelgrossen cystischen Tumor verwandelt. Beim Ausschälungsversuch platzt er, und es entleert sich eine trübe, eitrig seröse Flüssigkeit. Entfernung des rechten Adnextumors mit der Tubenecke. Toilette, Verschluss mit Etagennaht. Reconvalescenz durch Stichkanaleiterung etwas gestört und protrahirt. Am 22. 4. 99 geheilt entlassen.

Pathologischer Befund: Die linken Adnexe bestehen aus der linken Tubenecke mit der stark geschlängelten, knotig verdickten Tube, deren Aussenfläche mit kleinen abgerissenen Strängen reichlich bedeckt ist. Ihre Länge beträgt $12 \mathrm{~cm}$; ihr Fimbrienende ist gut entwickelt. Auf Druck entleeren sich aus dem abdominalen Ostium tubae gelblichkrümelige Massen. - Die rechte Tube ist von ihrer Tubenecke an stark greschlängelt, in 5 Windungen an- und nebeneinander gefaltet und mit Adhäsionen bedeckt. Ihr Umfang nimmt abdominalwärts bis auf $7 \mathrm{~cm}$ zu. Die Tube geht in eine apfelgrosse Cyste über, die bei der Operation einriss. Ihr liegt das Ovarium unten, vorn und medial wandständig an. Das Ovarium ist nicht wesentlich vergrössert, enthält mehrere bis erbsengrosse Cysten und ist mit dem Uterus, der Tube und der apfelgrossen Cyste durch zahlreiche Adbäsionen verbunden. Nachdem letztere vollends eröffnet ist, sieht man, dass das Fimbrienende der noch ca. $1 / 2 \mathrm{~cm}$ in sie frei hineinragenden Tube allseitig fest von ihr umschlossen wird, während die grossen, schön entwickelten Fimbrien sich an der Cystenwand fächerförmig ausbreiten, ohne aber an sie angewachsen zu sein, so dass sie in der mit Flüssigkeit gefüllten Cyste flottirt haben müssen. Aus dem durchgängigen Ostium abdominale tubae entleerte sich auf Druck gleich nach der Operation gelblicher Eiter. Die Cystenwand ist ca. $4 \mathrm{~mm}$ stark und zeigt eine körnig faserige, zerklüftete Innenfläche. Eine Communication zwischen dieser Cyste und einer der erwähnten kleinen Cystchen im Ovarium besteht nicht. Dio mikroskopische Untersuchung der Cystenwand zeigt, diss dieselbe aus drei Bindegewebsschichten besteht: Die äusserste hat wenig Kerne und führt straffes Bindegewebe; die mittlere Schicht ist 
Figur 3 .

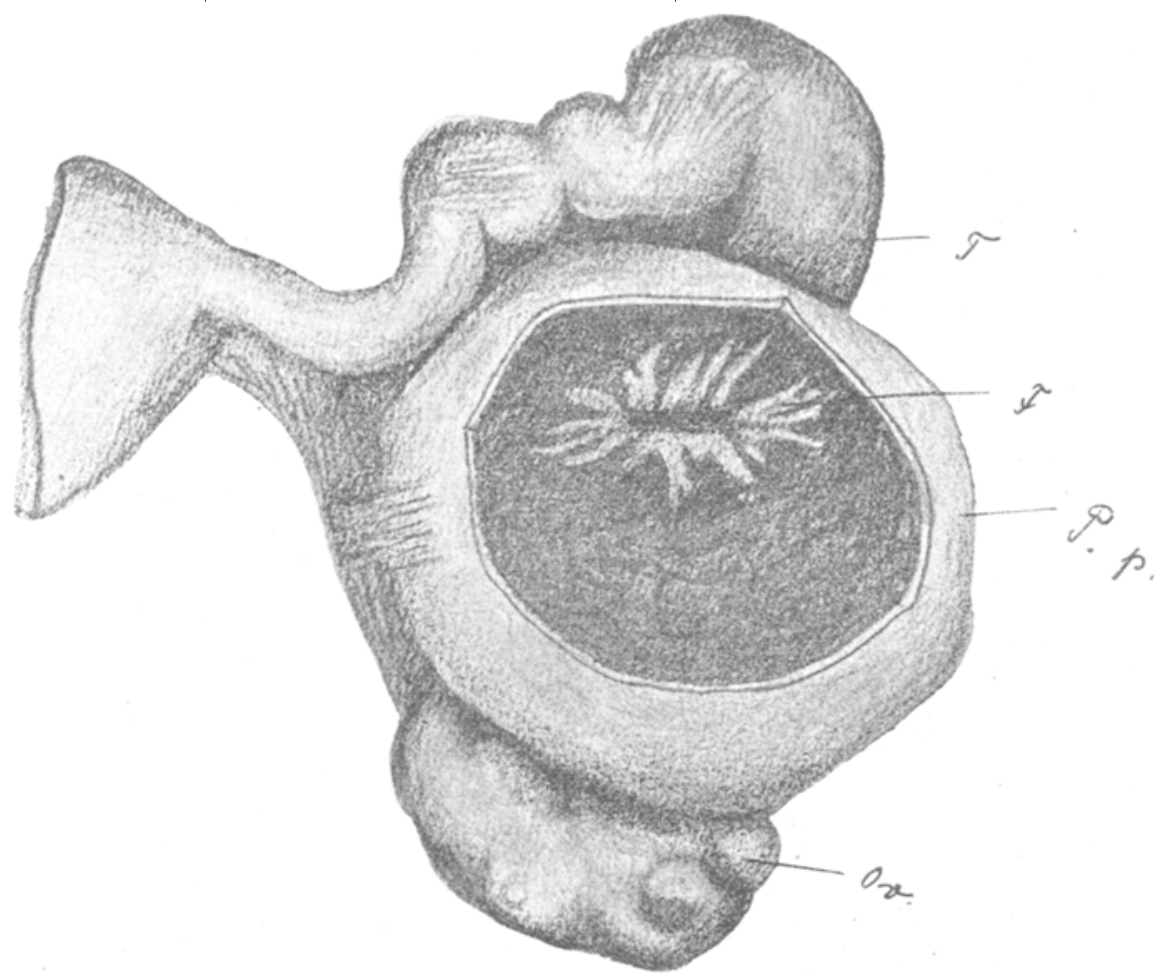

Fall K. Rechtsseitiger Abnextumor, veranschaulicht das Vorstadium einer Tuboovarialcyste durch die die Tube und das Ovarium verbindende "Pyocele peritubaria". Die Pyocele umgiebt das abdominale Tubenende, dessen Fimbrien in ibrem Lumen frei flottiren, während das Ovarium auf der anderen Seite der Pyocele angelagert ist.

T. Tube. -- F. Fimbrien. - P. p. Pyocele peritubaria. - Or. Ovarium.

etwas kernreicher mit einzelnen eingesprengten Leukocytenherden; die innerste besteht aus Granulationsgewebe. Fierstocksgewebe ist, wie ja auch nach dem makroskopischen Befunde zu erwarten war, nicht in der Cystenwand aufzufinden.

Wir haben es hier also mit einem Befunde zu thun, wie ihn auch Zahn, Waldstein, Zedel u. a., wie bereits erwähnt, gefunden haben. Die nicht verschlossene Tube mündet in eine apfelgrosse Cyste, deren Wand ihr Fimbrienende fest umschliesst. Das Ovarium liegt der Cyste wandständig an, der Tubenmündung geradeüber, bildet aber keinen wesentlichen Bestandtheil der Cyste. Es hat sich hier also um ein der Tube entstammendes Exsudat 
gehandelt, um das sich eine Pfannenstiel'sche "Pyocele peritubaria" als Abkapselungsmembran gebildet hat. Diese hat auch das Ovarium theilweise eingeschlossen und so Tube und Ovarium zu einem einheitlichen Adnextumor verbunden, der makroskopisch durchaus den Eindruck einer Tuboovarialcyste machte. Wäre hier noch eine Ovarialcyste in den Pyocelenraum hineingeplatzt, so wäre jene Art von Tuboovarialcyste entstanden, bei der die Fimbrien frei im Cysteninnern flottiren.

Eine weitere Stufe der Entwicklung zur echten Tuboovarialcyste, als wie sie uns der eben besprochene Fall bot, zeigt der nächste Befund. Hier ist nämlich sowohl "Pyocele peritubaria", wie Ovarialcyste vorhanden, jedoch die Einschmelzung der Scheidewand zwischen beiden noch nicht erfolgt.

Fall Wedekind. Die 45jährige Pat. tritt am 11.10.1898 in die Klinik ein. In ihrer Jugend war sie immer gesund. Vor 18 Jabren machte sie einen schweren Gelenkrheumatismus durch und klagte seitdem über Herzklopfen und Athemnoth. Ein Jahr später bekam sie Malaria. Vor 6 Jahren erlitt sie ein Trauma gegen den Unterleib und behauptet, seitdem Schmerzen und unregelmässige Blutungen gehabt zu haben. Zwei Jahre später kam sie „wegen Unterleibsentzündung" in ärztliche Behandlung. Vor zwei Jahren machte sie dann eine Pneumonie durch. Die unregelmässigen Blutungen verloren sich jedoch vor elf Monaten, als ihr ein Gebärmutterpolyp entfernt worden war. Seitdem bis zur Aufnahme regelmässig menstruirt. Seit etwa fünf Monaten bemerkte sie eine Abnahme ihres Körpergewichts, bis sich vor ca. drei Wochen in der rechten Bauchseite und der Magengegend Schmerzen einstellten, verbunden mit einem Gefühl des Vollseins im Leib und Brechreiz. Auch Fieber will sie gehabt haben. Seit drei Wochen ist die seitdem fällige Periode nicht eingetreten.

Status: Mittelgrosse, kränklich aussehende Frau, normal gebaut. Herz o. B. Lungen r. b. o. rauhes Inspirium, keine Geräusche. Innere Untersuchung: Enge Scheide; vom anteflectirten Uterus nicht abgrenzbar, fühlt man fast in der Mitte, aber etwas mehr links, einen ziemlich festen, ca. kindskopfgrossen Tumor. Untersuchung selr schmerzhaft. Am 13. 10. 1898 in Chloroformäthernarcose Laparotomie in der Linea alba. Nach der Eröffnung des Peritoneums durch Längsschnitt zeigt sich das Netz und nach seiner Lösung der Tumor so mit den Därmen und seiner Umgebung verwachsen, dass eine Orientirung zunächst nicht möglich ist. Der Uterus ist nicht zu fühlen. Erst mit Mühe gelingt es, den Tumor zunächst rechts, dann links etwas freizubekommen. Dabei platzt er und entleert übelriechenden, eitrigen Inhalt mit gallertigen Fetzen. Die Adhäsionen bluten stark; es wird provisorisch tamponirt. Nach Vorziehen des Tumors mit Cystenzangen zeigt sich, dass derselbe vom linken Ovarium und der Tube ausgebt. Der Uterus ist ganz nach rechts verlagert und lässt sich nicht in die Mitte bringen. Nach Abpräparirung der flächenhaft verwachsenen Blase und Isolirung der Geschwulst wird dieselbe nach einer Reihe von Unterbindungen hart am Uterus abgesetzt und entfernt. Die Blutung steht. - Die 
rechten Adnexe erweisen sich als stark geschrumpfte und atrophische Organe, hart am Uterus liegend und verwachsen. Von ihrer Entfernung wird abgesehen. Nach Reinigung der Bauchhöhle mit Tavel'scher Lösung und Austupfen wird durch eine Binde, die zum unteren Wundwinkel herausgeleitet wird, tamponirt und die Bauchwunde mit durchgreifenden Seidennähten geschlossen. Nach der einstündigen Operation sehr schlechter Puls. Trotz Excitantien und Kochsalzinfusion am nächsten Tage Exitus. Autopsie verboten.

Pathologischer Befund: Die linke Tube ist an einzelnen Stellen bis auf $12 \mathrm{~cm}$ Umfang verdickt, mit zahlreichen Adhäsionen bedeckt und in mehreren Windungen U-förmig gebogen und zwar so, dass in situ die Oeffnung des U medianwärts, das Knie lateralwärts lag. Zwischen einzelnen Adhäsionsmembranen, die von Windung zu Windung ziehen, sind einzelne Taschen und Buchten, die mit grünlichem, übelriechendem Eiter erfüllt sind und zum Theil bei der Operation einrissen. Die Tube geht allmälig, aussen nur durch eineseich te Einschnürung erkennbar in eine ca. hühnereigrosse, collabirte Cyste über, die bei der Operation einriss und die sich ihrerseits an einen überkindskopfgrossen Tumor anlegt, der mit den Därmen und der hinteren Beckenwand fest verwachsen war. Vom Ovarium ist makroskopisch nichts zu entdecken. Nachdem das Präparat aufgeschnitten ist, zeigt sich, dass die Tubenwandung enorm verdickt, das Lumen aber noch nicht verlegt ist. Durch ihre ca. hanfkorngrosse Abdominalöffnung gelangt man in die oben beschriebene hühnereigrosse Cyste, an deren Wand noch einige längliche, streifenartige Gebilde zu sehen sind, die um die Mündungsstelle der Tube radiär ausstrahlen und deswegen für stark veränderte, an der Wand angewachsene Fimbrien gehalten werden können, eine Vermuthung, welche die mikroskopische Untersuchang in der That bestätigte. Zwischen ihnen ist die Wand zerklüftet und macht den Eindruci einer Abscessmembran. Diese Cyste stösst an die ebenfalls oben erwähnte, über kindskopfgrosse Cyste und an eine ca. kirschgrosse, ebenfalls Eiter enthaltende, beiden wandständige Cyste an. Alle 3 Cysten stehen nicht mit einander in Communication. Die gemeinsame Scheidenwand zwischen der hühnereigrossen und der über kindskopfgrossen Cyste ist von ca. Zweimarkstückgrösse und stellenweise bloss $3 \mathrm{~mm}$ stark, so dass sich hier wohl ein Durchbruch vorbereitet haben mag, da die Wand sonst ca. $5 \mathrm{~mm}$ dick, stark zerklüftet und zerfasert und stellenweise mit dicken Borken eingedickten Eiters bedeckt ist. Nur an einer, der Oeffnung des Tuben-U gegenüberliegenden Stelle der grossen Cyste ist die Wand derselben in einer Flächenausdehnung von ca. Thalergrösse bis ca. $15 \mathrm{~mm}$ dick, und man gewahrt hier schon makroskopisch grössere Gefässe. Die mikroskop ische Untersuchung zeigt, dass alle Schichten der Tube kleinzellig infiltrirt und mit kleinen Abscessen durchsetzt sind. Die Wand der hübnereigrossen Cyste, in welche die Tube einmündet, besteht aus straffem Bindegewebe mit schichtweise concentrisch eingesprengter kleinzelliger Infiltration. Innen findet man Granulationsgewebe. Nur an den Stellen, wo makroskopisch sichtbar ander Wand sich die streifenähnlichen Gebilde radiär von der Tubenmündung ausstreckten, lässt sich in der That Fimbrienepithel, zum Theil auch noch schwache Muskelzüge nach- 
weisen. Die Wand der kirschgrossen, wandständigen Cyste zeigt ebenfalls typischen Abscessmembranbau ohne jedes Epithel. Die über kindskopfgrosse Cyste besteht in ihrer grössten Wandausdehnung ebenfalls aus Abscessmembran, innen stark zerklüftetes Granulationsgewebe tragend und nur in der bereits oben erwähnten thalergrossen Wandfläche, wo die Wanddicke bis $15 \mathrm{~mm}$ zunimmt, kann man noch allerdings sehr verändertes Ovarialgewebe nachweisen, in Form von Corpora fibrosa und einigen atretischen Follikeln.

Diese letzte Cyste dürfte daher als Ovarialeyste anzusprechen sein, während man in der hühnereigrossen Cyste mit Recht wird eine "Pyocele peritubaria" erblicken dürfen, an deren Innenwand die Fimbrien festgewachsen sind. Dic kirschgrosse Cyste ist jedenfalls ein abgekapselter Abscess, ebenfalls eine Folge der in der Anamnese bestehenden Peritonitis. Hier hätte bei längerem Bestande und bei einer Druckzunahme in der hühnereigrossen "Pyocele peritubaria", die jetzt immerhin noch $3 \mathrm{~mm}$ starke Zwischenwand zwischen ihr und der grossen Cyste zu Grunde gehen können, und 'wir hätten dann ebenfalls den Befund einer echten Tuboovarialcyste. In dieser hätten die Fimbrien jedoch nicht flottirt, sondern wären an der Wand angewachsen gewesen.

In dem folgenden Fall ist diese Communication zwischen Pyocele peritubaria und Ovarialcyste bereits erfolgt.

Fall Schaal. Die 38jährige Patientin war bis vor sechs Wochen regelmässig menstruirt. Sie ist seit 20 Jahren verheirathet und machte vor 17 Jahien ein normales Wochenbett durch. Seit 5-6 Jahren hatte sie zeitweise exacerbirende Schmerzen im Unterleibe, welche verschiedene Naturheilkundige (immer ohne Erfolg) zu vertreiben suchten. Seit 5-6 Wochen gesellten sich zu diesen Schmerzen auch noch heftigere Blutungen, so dass sie Herrn Professor Pfannenstiel aufsuchte.

Status: Grosse, sehr gealtert aussehende Frau von blasser Hautfarbe. Leib leicht gewölbt. Bauchdecken schlaff. Ziemlich weite Scheide, Uterus anteflectirt, hart und mittelgross. Zur linken Seite desselben liegt ein überfaustgrosser, derber Tunor, unbeweglich und auf Druck sehr schmerzhaft. - Am 14.11. 1900 wird die raginale Radicaloperation vorgenommen und der Uterus und die beiderseitigen, z. Th. sehr veränderten Adnexe entfernt. Prima intentio. Am 4. 12. 1900 wird Patientin geheilt entlassen.

Pathologischer Befund: Der Uterus ist metritisch vergrössert, bietet aber sonst nichts Besonderes. Die linken Adnexe sind zum Theil bei der Operation eingerissen. Die rechte Tube ist geschlängelt, die Fimbrien z. Th. eingekrempelt uod mit Adhäsionen bedeckt. Das rechte Ovarium ist etwas vergrössert. Auf der linken Seite geht die mit reichlichen Adbäsionsresten bedeckte Tube am abdominalen Ende in eine ca. apfelsinengrosse Cyste über, an deren entgegengesetztem Pole ein etwa hühnereigrosser Knollen von theilweise cystischer Beschaffenheit ansitzt.' Nachdem durch alle drei Gebilde ein Frontalschnitt hindurch gelegt ist, zeigt sich, dass die nicht verschlossene Tube in die 
ca. apfelsinengrosse Cyste einmündet. Diese selbst ist mit geruchlosem, dünnflüssigem, gelblichem Eiter erfüllt und communicirt ihrerseits wiederum durch eine ca. linsengrosse Oeffnung mit dem erwähnten, hühnereigrossen Körper. Im Gegensatz zu diesem, theilweise soliden Knollen besitzt die apfelsinengrosse Cyste eine überall gleichbleibende Wandstärke von $3-5 \mathrm{~mm}$. Die Innenfäche dieser Cyste zeigt ein feinkörniges Aussehen von gelber Farbe.

Wie die mikroskopische Untersuchung ergiebt, haben wir in dem hühnereigrossen Körper das mit mit vielen, z. Th. mit einander communicirenden Abscessherden durchsetzte Ovarium zu, erblicken, während die apfelsinengrosse Cyste den charakteristischen, oben beschriebenen Bau einer Pfannenstiel'schen "Pyocele peritubaria" aufweist. Das Fimbrienende wird von dieser umfasst, die Fimbrien selbst sind theilweise mit ihren abdominalen Flächen innerhalb dieser Cyste verklebt, theilweise flottiren sie auch frei.

Wir haben also hier folgenden Befund vor uns: Die nicht verschlossene Tube mündet in eine „Pyocele peritubaria". Wandständig an dieser liegt das vereiterte Ovarium, welches mehrere Cysten, darunter eine circa daumengliedgrosse; birgt. Von der Zwischenwand zwischen dieser und der Pyocele ist bereits ein linsengrosses Stück durch Druckatrophie zu Grunde gegangen. Wir haben somit hier eine echte Tuboovarialcyste vor uns, bei der das $Z$ wisehenglied einer Pyocele peritubaria überaus deutlich zu erkennen ist.

Die "Pyocele peritubaria" braucht natürlich durchaus nicht immer noch die ausgeprägte Jugendform einer abgerundeten, mehr oder weniger in sich abgeschlossenen Cyste zu zeigen, wie in den bisherigen Fällen, sondern sie kann, an sich klein und die Fimbrien grade umschliessend, auch noch einen Theil ihrer Wandung bei der Herstellung der Communication zwischen ihr und der Ovarialcyste eingebüst haben, zugleich den Theil der Fimbrien, der an dem zu Grunde gegangenen Abschnitt der Zwischenwand angewachsen war. Das lehrt folgender Fall, dessen Zustandekommen in Fig. 4 schematisch zum Ausdruck gebracht wird:

Fall Hoffmann. Die 31jährige Pat. ist seit ihrem 17. Lebensjahre alle 4 Wochen je 4 Tage lang regelmässig menstruirt; nur in letzter Zeit stellte sich die Regel 2-3 Tage früher ein. Vor 13 Jahren machte sie nach eirrer Geburt und $1 / 2 \mathrm{Jahr}$ später nach einem Abort normale Wochenbetten durch. Vor 3 Wochen erkrankte sie an profusen Diarrhöen, worauf sich heftige Schmerzen in der rechten Unterleibsseite einstellten, die so stark wurden, dass sie laut aufschreien musste.

Am 10. November $1899 \mathrm{kam}$ sie in die Behandlung des Herrn Professor Pfannenstiel, in der sie damals 8 Tage verblieb. Der 
Uterus stand damals beweglich in der Retroversio-flexio; rechts von ihm ein schlaffwandiger, schmerzender Tumor, der bis zur rechten Beckenschaufel reicht. Anormale Bestandtheile im Stuhl, die auf ein etwaiges Darmleiden hinweisen könnten, sind nicht nachzuweisen. - Patientin wurde damals hydrotherapentisch behandelt, kam aber nach 2 Monaten mit dem Befunde einer fixirten Retroflexio und beiderseitiger Adnextumoren wieder. Am 9. 1. 1900 Operation, bei der der rechte Adnextumor einreisst und eine trübe, fettig-glänzende, serös-eitrige Flüssigkeit austreten lässt. Diese wird sorgfältig ausgetupft; nach einigen Tagen Icterus und eine rechtsseitige Pneumonie. Am 17. 2. wird Pat. geheilt entlassen.

Pathologischer Befund: Präparat besteht aus dem ziemlich hoch supravaginal abgesetzten Uterus und beiden z. Th. zu Tumoren verwandelten Adnexen. Die linke Tube ist ca. $10 \mathrm{~cm}$ lang und stark geschlängelt und erweitert sich allmälig wurstförmig bis zu einem Umfang von $6 \mathrm{~cm}$, so dass derselbe in der Höhe des Ostium abdominale dem des etwa walluussgrossen Ovariums gleichkommt. Die Fimbrien sind etwas einwärts gestülpt und lassen das Ostium abdominale für eine Fingerspitze durchgängig zwischen sich. Von ihrer Aussenfläche ziehen nach dem Ovarium adhäsive Stränge und Pseudomembranen. - Die rechte Tube ist am uterimen Ende von normaler Dicke, abdominalwärts aber bis zu Daumendicke zunehmend, stark geschlängelt und nach einem Verlaufe von $13 \mathrm{~cm}$ in einen hühnereigrossen Tumor übergehend. Die einzelnen Windungen, die sich $\mathrm{z}$. Th. nebeneinander gelagert haben, sind mit Adhäsionsresten allseitig bedeckt. Nachdem nun der hühnereigrosse, cystische Tumor eröffnet ist, zeigt sich, dass sich aus dem offenen Tubenlumen eitriges Secret in die Cyste entleeren lässt. Jedoch sind makroskopisch Fimbrien nicht zu sehen. Die Abdominalöfinung

Figur 4.

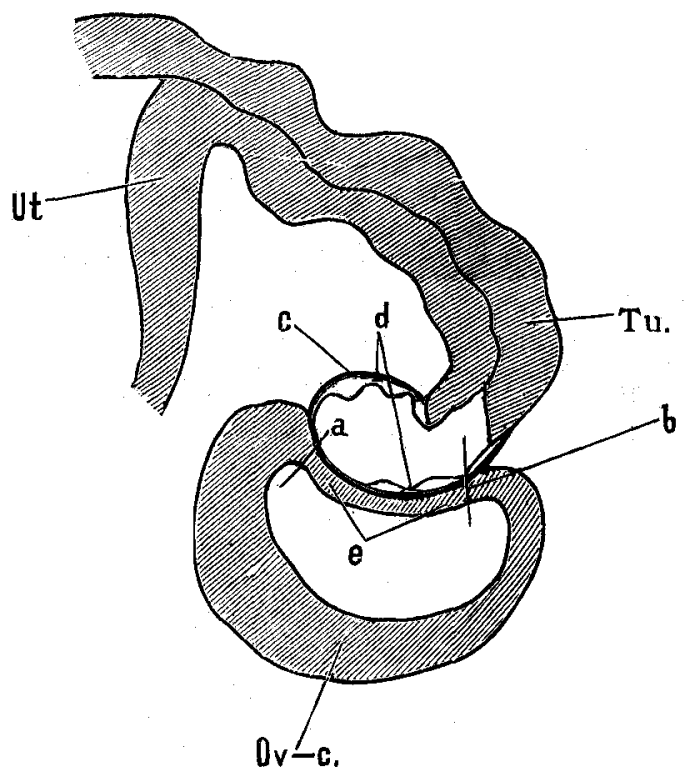




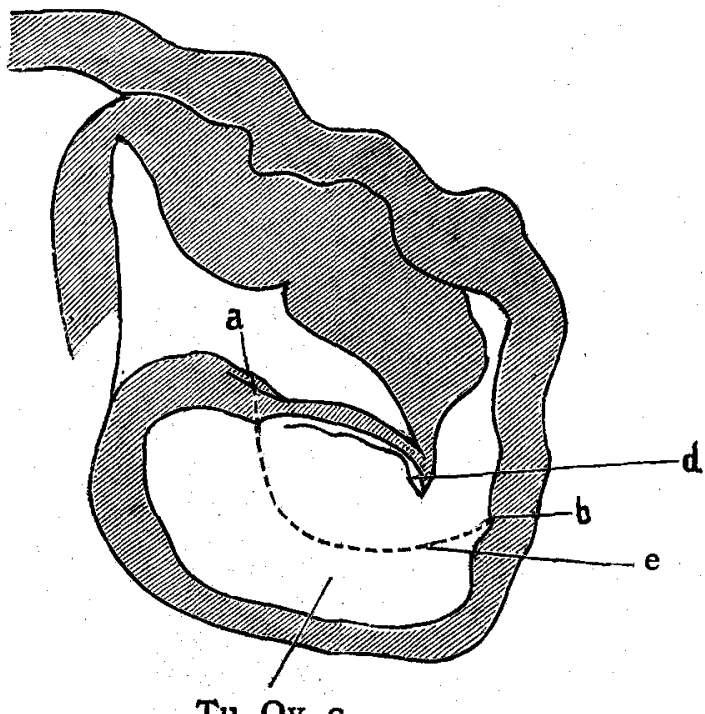

Tu.-Ov. c.

Adnextumor, veranschaulicht das Zustandekommen einer Tuboovarialcyste durch das Verkleben einer Ovarialeyste mit einer "Pyocele peritubaria". Das Fimbrienende der Tube wird umgeben von der Pyocele, an deren Wand die Fimbrien angewachsen sind. An die Pyocele hat sich eine Ovarialcyste in breiter Ausdehnung angelagert. Ihre Scheidewand von $a-b$ geht durch Druckatrophie zu Grunde, und so ist es möglich, dass man in der fertigen Tuboovarialcyste nur einen Theil der Fimbrien (an der oberen Cystenwand angewachsen) wiederfindet.

Ut. Uterus. - Tu. Tube. - c. Pyocele peritubaria. - d. Fimbrien. e. Zwischenwand. - Ov. c. Ovarialcyste.

der Tube erweist sich als wenige $\mathrm{mm}$ im Lumen haltender Spalt, in welchem man die feine Zeichnung der Tubenfalten deutlich erkennen kann. Die Innenwand der Cyste bietet das Bild einer körnigen Abscessmembran. Ihre Wandstärke schwankt zwischen $3-5 \mathrm{~mm}$. Makroskopisch ist Ovarialgewebe nicht zu sehen. Jedoch ergiebt die mikroskopische Untersuchung (vergl. Fig. 5), dass in der Cystenwand fast überall Ovarialgewebe nachweisbar ist, bestehend aus einigen atretischen Follikeln und vor Allem aus Corpora fibrosa. Nur in einem kleinen Bezirk nahe der Tubenmündung (in der Fig. 5 von $a-b$ ) findet sich in der Wand der Cyste kein früheres Ovarialgewebe. Dieser Bezirk hat etwa die Grösse eines Zehnpfennigstückes und ist dadurch ausgezeichnet, dass sich an der Cystenimnenffäche auch noch Fimbrien nachweisen weisen lassen, während die andere directe Umgebung der Tubenmündung Fimbrien, die man doch auch bier vermuthen muss, nicht aufweist. Die Innenfläche der Cyste ist epithellos. stark zerfaeert und als Corpus-luteum-Cyste aufzufassen. 
Figur 5 ,

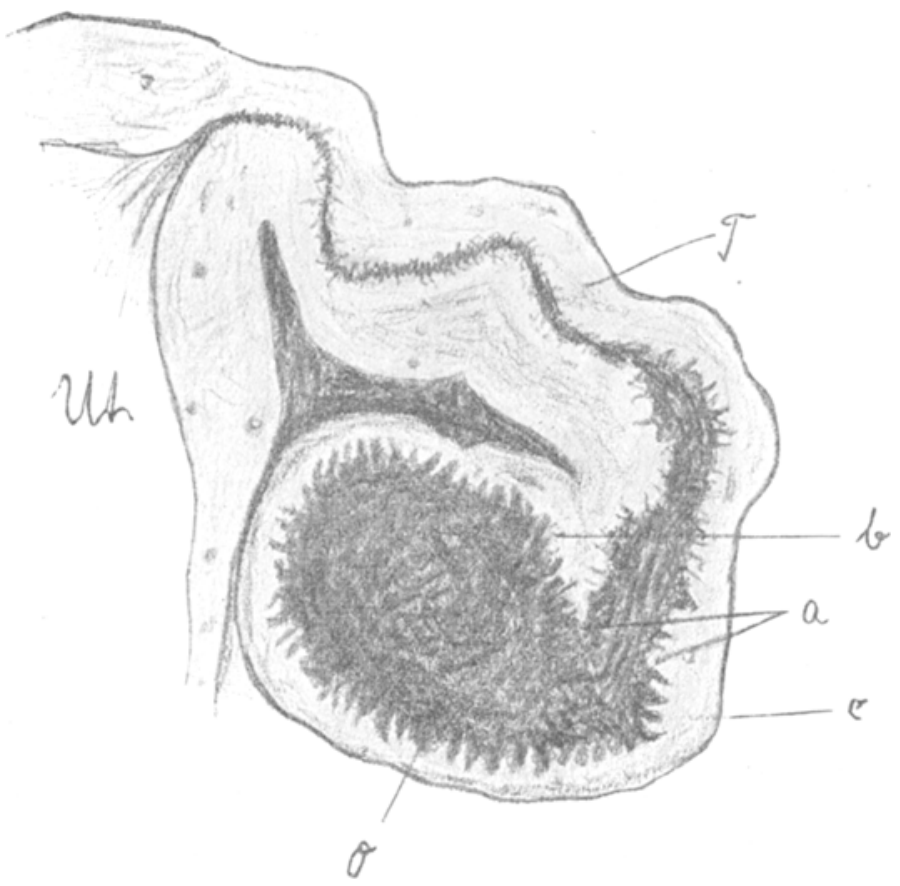

Tuboovarialcyste nach einem in Fig. 4 schematisch dargelegten Vorgang entstanden (Fall H.). Von $a-b$ ist im Cysteninnern allein noch Fimbriengewebe nachweishar, nicht aber in $\mathrm{c}$.

Ut. Uterus. - T. Tube. - 0. Ovarialcyste.

Offenbar ist dieser Befund so zu erklären: Die aus der Tube hervorquellende Eitermenge und das sich an dem Ostium abdominale sammelnde Exsudat war nicht sehr gross, in Folge dessen auch nicht die abkapselnde "Pyocele peritubaria", an deren Innenwand sich die Fimbrien anlegten und anwuchsen. Nun bestand $\mathrm{zu}$ gleicher Zeit eine Ovarialeyste; beide berührten sich und zwar so, dass ein grosser Theil der Pyocelenwand sich mit den angewachsenen Fimbrien an der Scheidewandbildung betheiligte (Fig. 4). Diese Scheidewand atrophirte nun mit den zugehörigen Fimbrien. Die jetzt einkammerige Cyste dehnte sich durch den Inhalt, und glich die beiden verschiedenen Cystenwölbungen aus. So kommt es, dass wir jetzt an der Wandstelle, wo die Tube in die Cyste einmündet, nur noch an einem Kreisabschnitt neben der Mündungsstelle Fimbrienreste finden. 
Fasse ich nun noch einmal am Schlusse meiner Arbeit die Ergebnisse dieser vorliegenden Untersuchungen zusammen, so lassen sich folgende Sätze aufstellen:

1. Eine congenitale "Ovarialtube" ist beim Menschen bisher noch nicht mit Sicherheit festgestellt worden.

2. Die Mehrzahl der Tuboovarialcysten ist dadurch entstanden, dass eine Sactosalpinx und eine Ovarialyste zusammen verklebten und dass ihre Zwischenwand später durch Druckatrophie zu Grunde ging; von Fimbrien ist in diesen Fällen meist nichts mehr nachzuweisen, da sie bei der Atrophie der Scheidewand ebenfalls verloren gingen.

3. Die Entstehung einer Tuboovarialcyste auf dem Wege einer Hämatocelenbildung ist denkbar, steht aber mit der Häufigkeit der doppelseitigen Befunde und dem negativen Blutnachweise nicht in Einklang.

4. Der Befund bei gewissen Tuboovarialcysten, in deren. Inneren die Fimbrien entweder frei flottiren oder an der Wand angewachsen sind, ist nach Pfannenstiel in der Weise zu erklären, dass ursprünglich sich um das der Tube entstammende Exsudat eine "Pyocele peritubaria" unter Bildung einer sich organisirenden Abkapselungsmembran etablirt hat, welche, da sie das Tubenende allseitig umschlicsst, das Zwischenglied zwischen der Tube und dem cystischen Ovarium darstellt. Nach Schwund der Wand zwischen Pyocelenhöhle und Ovarialcyste entsteht die Tuboovarialcyste. In frühen Stadien findet man noch Eiter in dem cystischen Raum; später pflegt derselbe durch seröse Flüssigkeit ersetzt zu werden. 